\title{
Polymer viscosifier systems with potential application for enhanced oil recovery: a review
}

\author{
Kelly Lúcia Nazareth Pinho de Aguiar ${ }^{1, *}$ (D), Luiz Carlos Magalhães Palermo ${ }^{1}$ and Claudia Regina Elias Mansur ${ }^{1,2}$ \\ ${ }^{1}$ Federal University of Rio de Janeiro, Institute of Macromolecules, Horácio Macedo Avenue, 2030-Block J, \\ 21941-598 Rio de Janeiro, Brazil \\ ${ }^{2}$ Federal University of Rio de Janeiro, Program of Materials and Metallurgy Engineering/COPPE, \\ Horácio Macedo Avenue, 2030-Block F, 21941-598 Rio de Janeiro, Brazil
}

Received: 5 January 2021 / Accepted: 23 July 2021

\begin{abstract}
Due to the growing demand for oil and the large number of mature oil fields, Enhanced Oil Recovery (EOR) techniques are increasingly used to increase the oil recovery factor. Among the chemical methods, the use of polymers stands out to increase the viscosity of the injection fluid and harmonize the advance of this fluid in the reservoir to provide greater sweep efficiency. Synthetic polymers based on acrylamide are widely used for EOR, with Partially Hydrolyzed Polyacrylamide (PHPA) being used the most. However, this polymer has low stability under harsh reservoir conditions (High Temperature and Salinity HTHS). In order to improve the sweep efficiency of polymeric fluids under these conditions, Hydrophobically Modified Associative Polymers (HMAPs) and Thermo-Viscosifying Polymers (TVPs) are being developed. HMAPs contain small amounts of hydrophobic groups in their water-soluble polymeric chains, and above the Critical Association Concentration (CAC), form hydrophobic microdomains that increase the viscosity of the polymer solution. TVPs contain blocks or thermosensitive grafts that self-assemble and form microdomains, substantially increasing the solution's viscosity. The performance of these systems is strongly influenced by the chemical group inserted in their structures, polymer concentration, salinity and temperature, among other factors. Furthermore, the application of nanoparticles is being investigated to improve the performance of injection polymers applied in EOR. In general, these systems have excellent thermal stability and salinity tolerance along with high viscosity, and therefore increase the oil recovery factor. Thus, these systems can be considered promising agents for enhanced oil recovery applications under harsh conditions, such as high salinity and temperature. Moreover, stands out the use of genetic programming and artificial intelligence to estimate important parameters for reservoir engineering, process improvement, and optimize polymer flooding in enhanced oil recovery.
\end{abstract}

\section{Introduction}

Oil is the most important energy source in the world and its production is crucial for the global economy. With industrial development and population growth, oil demand tends to increase by about $1.5 \%$ a year on average. Furthermore, it is estimated that petroleum will be the largest contributor to world energy needs for at least the next two decades (El-Hoshoudy et al., 2017a; Firozjaii and Saghafi, 2019; International Energy Agency, 2019; Kamal et al., 2015a).

Crude oil can be obtained from three different processes, namely primary, secondary and enhanced oil recovery. Primary recovery involves extracting crude oil using the natural energy from the reservoir, whereby it is possible to recover about $5.0 \%$ to $15.0 \%$ of the initial reserve.

\footnotetext{
* Corresponding author: kellypinho@ima.ufrj.br
}

Secondary recovery involves injection of immiscible fluids such as water, gas (or both) to displace the oil without changing the reservoir's natural conditions, enabling an additional recovery factor of $10.0 \%$ to $30.0 \%$. Enhanced Oil Recovery (EOR) methods allow maximizing oil production with additional production of approximately $10.0 \%$ to $35.0 \%$ over secondary recovery, using unconventional techniques involving injection of chemicals, thermosensitive fluids, microbiological agents and miscible gases, among others (Firozjaii and Moradi, 2018; Mogollón et al., 2019; Rellegadla et al., 2017; Thomas et al., 2012).

Due to the increased demand for oil, the lower discovery of new reservoirs and the large number of mature oil fields, i.e., those that have already gone through their production plateau where output is now declining, EOR methods are being increasingly applied since they allow changing the displacement mechanism in the reservoir rock to boost the 
oil recovery factor (Abidin et al., 2012; Bai et al., 2015; ElHoshoudy et al., 2017a). Among the EOR techniques, chemical Enhanced Oil Recovery (cEOR) stands out, which consists of the application of polymers, surfactants, alkalis or mixture of these components. In this category, polymer flooding is generally considered to be the most promising technique, representing about $77.0 \%$ of cEOR (Rellegadla et al., 2017; Sheng et al., 2015; Thomas, 2016).

Polymer application can increase the viscosity of the injection fluid, decreasing the water-oil mobility ratio and water breakthrough, improving the sweep efficiency and thereby increasing the oil recovery factor (Jung et al., 2013; Rellegadla et al., 2017; Sedaghat et al., 2013).

Several polymers can be applied in cEOR, of which acrylamide-based polymers stand out due to their wide availability and relatively low cost. Of them, Partially Hydrolyzed Polyacrylamide (PHPA) is used most for this purpose (Abidin et al., 2012; Data et al., 2018; Firozjaii and Saghafi, 2019). PHPA has high viscosifying power due to its high molecular weight and the electrostatic repulsion of the carboxylate groups present in its structure, which provide a higher hydrodynamic volume in solution, increasing the viscosity of the polymeric solution (Kamal et al., 2015a; Leblanc et al., 2015; Li et al., 2017).

However, PHPA poses stability problems under harsh reservoir conditions (high salinity and temperature), especially in the presence of divalent cations, where the polymer solution's viscosity decreases considerably, reducing the polymer flooding efficiency. Thus, the alternatives to overcome these limitations of PHPA are the use of Hydrophobically Modified Associative Polymers (HMAPs), Thermo-Viscosifying Polymers (TVPs) and nanoparticles (Abdullahi et al., 2019; Chen et al., 2013; Data et al., 2018; Gbadamosi et al., 2019a; Liu et al., 2017a; Sarsenbekuly et al., 2017a; Wang et al., 2011).

Hydrophobically modified associative polymers consist of water-soluble macromolecules modified with small fractions of hydrophobic chains. These have a Critical Association Concentration (CAC), so that amounts close to the CAC cause the formation of hydrophobic microdomains that increase the viscosity of the polymer solution (Data et al., 2018; El-Hoshoudy et al., 2017a; Kamal et al., 2015a). TVPs have blocks or grafts with Low Critical Solution Temperature (LCST), incorporated in the main water-soluble chains of the polymer, having the common behavior of macromolecules at room temperature. But above the association temperature $\left(T_{\text {ass }}\right)$, thermosensitive sequences self-assemble and form microdomains, greatly increasing the polymer solution's viscosity (Cao et al., 2015; Chen et al., 2013; Li et al., 2017; Wang et al., 2010).

The main aim of this review is to summarize the contemporary research involving the various polymer flooding techniques for enhanced oil recovery and present the benefits of applying HMAPs, TVPs and nanoparticles to improve the oil recovery factor under harsh reservoir conditions (high salinity and temperature). In addition, to present the use of artificial intelligence to estimate important parameters for reservoir engineering and to improve the polymer flooding process.

\section{Polymer flooding}

Polymer flooding is a chemical enhanced oil recovery method in which water-soluble polymers with high molecular weight are added to the injection fluid to increase its viscosity and thus to reduce its mobility ratio, providing greater sweep efficiency and an increase in oil recovery. Polymer injection is considered one of the most promising EOR techniques due to its good efficiency and technical and economic viability (Choi et al., 2016; Data et al., 2018; Gbadamosi et al., 2019b; Pope, 2011; Rellegadla et al., 2017; Stavland et al., 2010).

This method was proposed for EOR in the mid-1960s and since then many studies and applications have been implemented in several oil fields, both in pilot and commercial scale. Currently, this method's importance is growing in the global energy market (Delamaide et al., 2014; Khamis et al., 2018; Mahran et al., 2018; Manrique et al., 2017). According to Gbadamosi et al. (2019b), polymer flooding has been successfully applied in many fields, such as Daqing in China, Tambaredjo in Suriname, Eastern Bodo and Pelican Lake reservoirs in Canada, and Marmul in Oman, among others. Of these examples, the application of polymer flooding in the Danqing field stands out based on the results, where output increased by up to 300000 barrels per day (Cheraghian and Hendraningrat, 2016).

According to $S N F$ (2020), polymer flooding presents a satisfactory Utility Factor (UF), which consists of the relationship between the amount of polymer (in $\mathrm{kg}$ ) needed to recover an additional barrel of oil, characterizing the method's economic feasibility. Studies of pilot plant projects have indicated that the average UF for polymer injection is one ton of polymer to increase the oil production by 80 barrels. Thus, polymer flooding can increase oil production by about $20 \%$, at a cost of $\$ 3$ to $\$ 6$ per extra barrel of oil.

One of the most important parameters for this cEOR process is the water-oil mobility ratio, which represents the effects of relative permeability and oil and water viscosity on the mobility of these fluids, following Darcy's Law (Eq. (1)), where $M$ is the mobility ratio between water-oil; $\lambda_{\mathrm{w}}$ is the mobility of the aqueous phase; $\lambda_{\mathrm{o}}$ denotes the mobility of the oil phase; $\mu_{\mathrm{w}}$ is the viscosity of water; $\mu_{\mathrm{o}}$ is the viscosity of the oil; $k_{\mathrm{w}}$ represents the relative permeability of the aqueous phase; and $k_{\mathrm{o}}$ is the relative permeability of the oil phase (El-Hoshoudy et al., 2019; Stavland et al., 2010):

$$
M=\frac{\lambda_{w}}{\lambda_{o}}=\frac{k_{w} / \mu_{w}}{k_{o} / \mu_{o}}=\frac{k_{w} \cdot \mu_{o}}{k_{o} \cdot \mu_{w}} .
$$

The application of polymers promotes an increase in injection water viscosity $\left(\mu_{\mathrm{w}}\right)$ and a decrease in the relative permeability of the aqueous phase $\left(k_{\mathrm{w}}\right)$ in the reservoir. Hence, the mobility ratio decreases $(M \leq 1)$ and causes a uniform front flow, acting as a piston to push the oil into the producing well, thus increasing the sweep efficiency and the oil recovery factor (Fig. 1b) (Abidin et al., 2012; Ferreira and Moreno, 2020; Kamal et al., 2015a; Thomas, 2016). On the other hand, water flooding increases the 


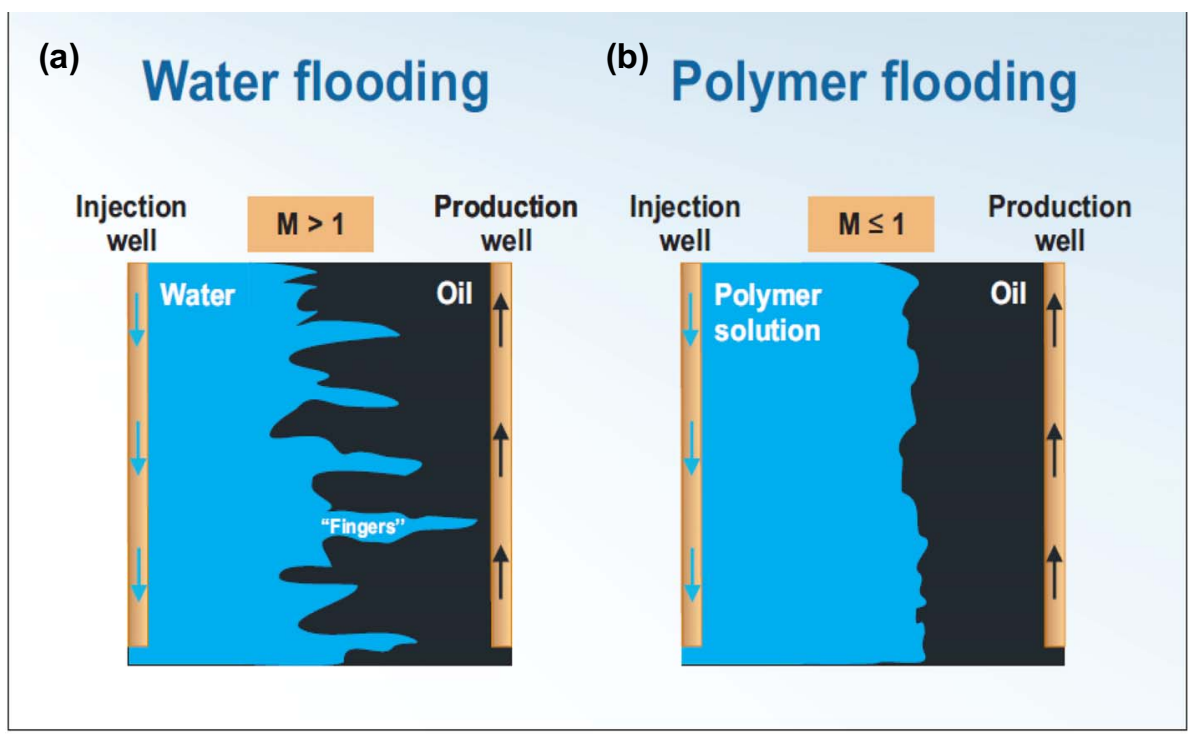

Fig. 1. Comparison between water flooding and polymer flooding, where: (a) water flooding forms "fingers" and decreases sweep efficiency, and (b) polymer flooding standardizes the front flow and increases the sweep efficiency.

mobility ratio $(M>1)$ due to the lower viscosity of the injected fluid, forming "fingers" in the reservoir, failing to displace a large amount of recoverable oil and reducing the sweep efficiency (El-Hoshoudy et al., 2019; Kamal et al., 2015a; Rellegadla et al., 2017; Fig. 1a).

Another important factor for the mobility ratio is the Buckley-Leverett model, which describes the frontal advance of immiscible displacement, such as the displacement of oil by the action of water, in a linear one-dimensional or almost one-dimensional reservoir, introducing a discontinuity of saturation. In this way, the displacement of the front-advance in fractional flow can be described according to equation (2), where $S_{\mathrm{D}}$ corresponds to the saturation of the displacement fluid; $\theta$ is the displacement time; $u$ represents the distance along the flow path; $q_{\mathrm{t}}$ characterizes the total rate of fluid flow through the section; $\mathrm{A}$ is the section area; $\Phi$ is the porosity; and $f_{\mathrm{d}}$ corresponds to the fractional flow of the displacement fluid (Buckley and Leverett, 1942; Dashtbesh et al., 2021; Guérillot et al., 2020):

$$
\left(\frac{\partial u}{\partial \theta}\right)_{S_{\mathrm{D}}}^{0}=\frac{q_{\mathrm{t}}}{A \Phi}\left(\frac{\partial f_{\mathrm{D}}}{\partial S_{\mathrm{D}}}\right)_{\theta}^{0}
$$

Other important parameters for polymer flooding are the resistance factor $\left(R_{\mathrm{F}}{ }^{*}\right)$ and the residual resistance factor $\left(R_{\mathrm{RF}}\right)$, which are obtained from oil recovery simulation tests in rock plugs (core flooding) and can indicate the efficiency of the oil displacement process. $R_{\mathrm{F}}{ }^{*}$ corresponds to the ratio between the brine mobility rate and polymer solution mobility (Eq. (3)). $R_{\mathrm{RF}}$ refers to the ratio between brine permeability before the polymer injection process and after the polymeric solution has flowed through the rock plug (Eq. (4)) (Ferreira and Moreno, 2020; Leiting et al., 2010; Maia et al., 2009; Wang et al., 2018a):

$$
R_{F^{*}}=\frac{\lambda_{\text {brine }}}{\lambda_{\text {polymer solution }}},
$$

$$
R_{\mathrm{RF}}=\frac{k_{w \text { (before polymer flooding) }}}{k_{w \text { (after polymer flooding) }}} .
$$

The polymer flooding technique can be applied in several sandstone and carbonate fields in order to increase oil recovery (Ferreira and Moreno, 2020). According to Wei and Romero-Zerón (2014), injection of polymers is not recommended for reservoirs containing oil with viscosity higher than $200 \mathrm{mPa}$ s, because under these conditions it would be necessary to add a very high concentration of polymer, which is not economically feasible. Furthermore, the high viscosity of the solution could pose fluid injectivity problems in the reservoir. Thus, the polymer concentration generally ranges from 1000 to $3000 \mathrm{ppm}$ (Kamal et al., 2015a; Wei and Romero-Zerón, 2014).

According to $\mathrm{Wu}$ et al. (2012), the candidate polymer for polymer flooding application needs to have certain characteristics, such as good solubility in brine; adequate viscosifying power even at low concentrations; low adsorption on the rock surfaces; and mechanical, chemical and thermal stability under reservoir conditions. Furthermore, the efficiency of using polymer solutions depends on several factors, such as concentration and polymer structure, salinity, temperature, $\mathrm{pH}$, reservoir rock type and oil viscosity, among others (Jung et al., 2013).

The polymers evaluated for polymer flooding are classified into two major groups: synthetic polymers and biopolymers (Firozjaii and Saghafi, 2019; Gbadamosi et al., 2019b; Rellegadla et al., 2017; Sheng et al., 2015).

In the class of synthetics, the highlights are Acrylamidebased polymers (AM), with Partially Hydrolyzed Polyacrylamide (PHPA) being used most for cEOR due to its low cost, excellent viscosity properties and good physicochemical characteristics (Abidin et al., 2012; Mahran et al., 2018; Stavland et al., 2010). Many research groups have conducted studies of the application of PHPA for polymer flooding (Choi et al., 2014, 2016; Clark et al., 2015; 
Gao, 2011, 2013; Hashmet et al., 2014a, b; Khamis et al., 2018; Levitt et al., 2013; Manichand et al., 2013; Oliveira et al., 2019; Rostami et al., 2018b; Zhang et al., 2011a).

The high viscosifying power of PHPA is directly related to its molecular weight and degree of hydrolysis, which typically ranges from $10 \%$ to $35 \%$. As the degree of hydrolysis increases, more carboxylate groups become available, increasing the number of negative charges with mutual electrostatic repulsion, generating higher hydrodynamic volume in solution, with consequent increase in viscosity (Ma et al., 2015; Mahran et al., 2018; Nwidee et al., 2016).

However, a very high degree of hydrolysis can cause greater sensitivity to the salinity of the medium, since in brines containing divalent cations, mainly $\mathrm{Mg}^{2+}$ and $\mathrm{Ca}^{2+}$, precipitation occurs due to the complexation between the divalent cations and PHPA carboxylate groups, decreasing the solubility in water. Another problem is the poor performance of PHPA in high-temperature reservoirs. This favors thermal hydrolysis of the polymer chains' amide groups, generating more carboxylate groups and increasing the susceptibility to precipitation in high-salinity conditions (El-Hoshoudy et al., 2019; Oliveira et al., 2019; Wever et al., 2011).

Besides acrylamide-based synthetic polymers, biopolymers are another class of macromolecules used for $\mathrm{cEOR}$, especially Xanthan Gum (XG) (Jang et al., 2015; Kamal et al., 2015a; Rangel et al., 2012; Wei and Romero-Zerón, 2014).

Xanthan gum is a water-soluble polysaccharide with high molecular weight produced from the fermentation of Xanthomonas campestris bacteria. Due to its rigid chain, XG has low sensitivity to salinity and hardness, higher thermal stability in high-temperature reservoirs and greater shear strength than polyacrylamides (Ghoumrassi-Barr and Aliouche, 2016; Nwidee et al., 2016).

Thus, several studies have been published on the application of this biopolymer in cEOR (Ghoumrassi-Barr and Aliouche, 2016; Jang et al., 2015; Moura and Moreno, 2019; Wei et al., 2014; Xu et al., 2013). However, xanthan gum has high sensitivity to degradation by microorganisms, higher production cost than PHPA, and high risk of blocking the rock pores due to the high content of cellular debris, restricting its use in large-scale field applications (Firozjaii and Saghafi, 2019; Rostami et al., 2018b; Wei et al., 2014).

To minimize these limitations of the major synthetic polymers and biopolymers used for EOR, hydrophobically modified associative polymers and thermo-viscosifying polymers have been developed (Data et al., 2018; El-Hoshoudy et al., 2017a; Kamal et al., 2015b; Li et al., 2017; Liu et al., 2017a; Sarsenbekuly et al., 2017a; Wang et al., 2011). The main studies performed and the evolution of research focused on the application of these polymers are presented in Sections 3 and 4, respectively.

\section{Hydrophobically Modified Associative Polymers (HMAPs)}

Hydrophobically Modified Associative Polymers (HMAPs) have been developed to improve the performance of injection polymers in reservoirs with high salinity and temperature. HMAPs compose a class of water-soluble macromolecules formed by inserting small amounts of hydrophobic groups into a water-soluble polymeric chain. They are produced by copolymerization reactions of a hydrophobic monomer with a hydrophilic one or by grafting these groups on the water-soluble polymeric chain. To ensure solubility in aqueous media, the concentration of these hydrophobic fractions must be less than $5 \% \mathrm{w} / \mathrm{v}$ (El-Hoshoudy et al., 2017a; Kamal et al., 2015a; Liu et al., 2017a; Mahran et al., 2018; Zou et al., 2013).

The viscosity properties of HMAPs are directly related to the hydrophobic association effect of the groups inserted in the main chain, and the rheological behavior in solution is determined by the CAC of these polymers. Thus, below the CAC (diluted regime) intramolecular interactions of hydrophobic groups in solution are favored, slightly increasing the system's viscosity. At concentrations above the CAC (semi-diluted regime), intermolecular associations dominate intramolecular ones, forming a network structure, resulting in a significant increase in the viscosity of the polymeric solution (Afolabi et al., 2019; Azad et al., 2018; Bai et al., 2018; Zhang et al., 2015). Figure 2 depicts the mechanism of HMAPs' action, with increased viscosity as a function of polymer concentration.

The first hydrophobically modified associative polymers were produced by Dubin and Strauss (1967) by copolymerization of alkyl vinyl ether with maleic acid. However, the application of these systems in EOR was patented by Landoll (1985), based on modified Hydroxyethylcellulose (HEC) with long alkyl chains containing from 8 to 25 carbon atoms.

In 2007, HMAPs were tested on a pilot scale in the Bohai Bay field in China under severe salinity conditions. The results were a $25000 \mathrm{~m}^{3}$ increase in oil production and a $41 \%$ decrease in water production, indicating promise as EOR additives (Zhou et al., 2007). Since then, several researchers have conducted studies of the production and application of HMAPs for polymer flooding (Data et al., 2018; Gou et al., 2015; Han et al. 2018; Leiting et al., 2010; Liu et al., 2017a; Wang et al., 2012; Xie et al., 2019; Zhong et al., 2009).

Zhong et al. (2009) synthesized a hydrophobically modified acrylamide-based terpolymer with sodium 2-acrylamide-2-methylpropane sulfonate (NaAMPS) and 2-Vinyl Naphthalene (VN), and evaluated the stability of the polymer solution during 90 days at $80^{\circ} \mathrm{C}$ with brine containing sodium chloride $(\mathrm{NaCl})$. They found that the HMAP tested presented good stability, maintaining $88.3 \%$ of initial viscosity. This behavior can be explained by incorporation of $\mathrm{VN}$ chains in the terpolymer, since the presence of aromatic groups increased the chain stiffness and avoided hydrolysis of the amide groups at high temperatures, minimizing polymer degradation.

Maia et al. (2009) performed a comparative study between polyacrylamide and acrylamide copolymer with $\mathrm{N}-\mathrm{N}$-dihexylacrylamide, evaluating the effect of salinity on their rheological behavior and the potential applicability of these systems in EOR from core flooding experiments in a porous medium. They found that the addition of $\mathrm{NaCl}$ 


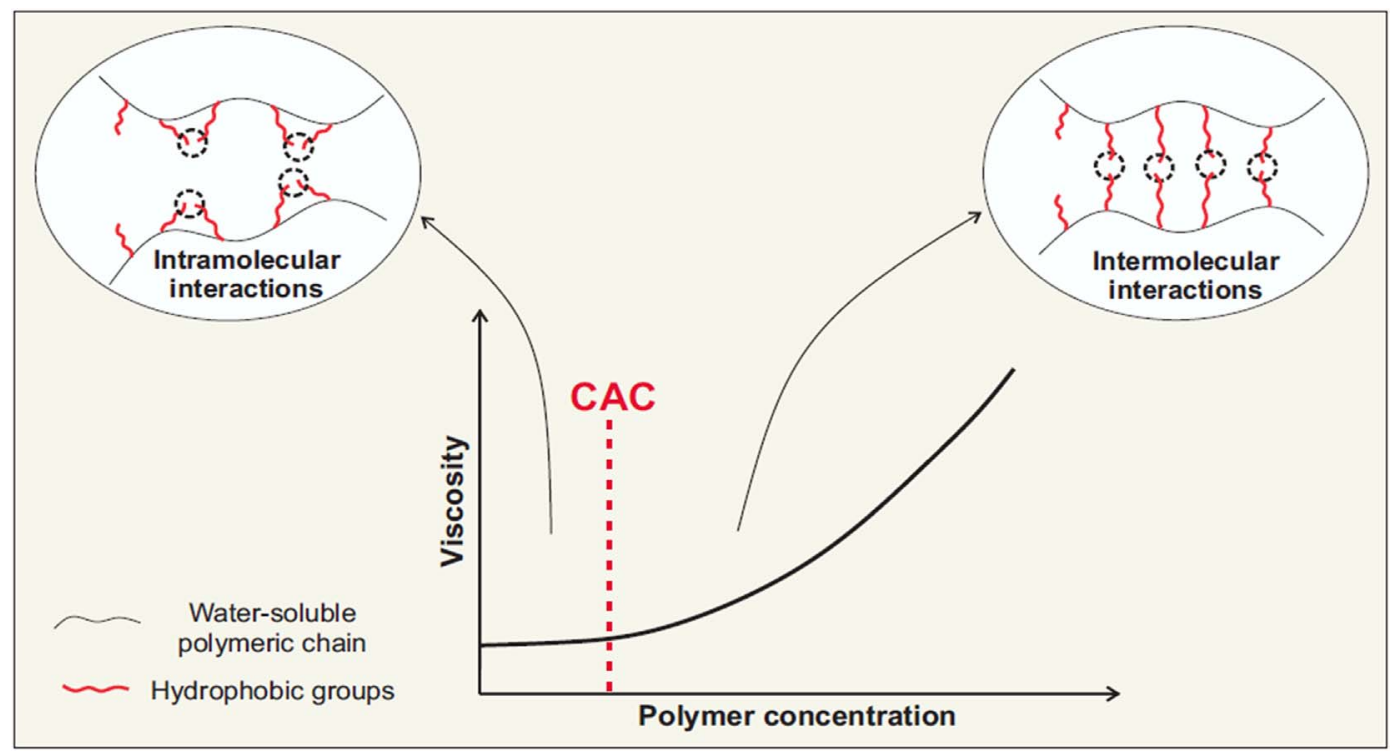

Fig. 2. Mechanism of HMAPs' action on polymer solution viscosity as a function of concentration.

promoted an increase in viscosity of the HMAP, and the $F_{\mathrm{R}}{ }^{*}$ of the solution of the copolymer was greater than that of commercial PHPA, indicating this copolymer as a promising agent to improve sweep efficiency. The comparison of HMAP and PHPA regarding resistance factor was also studied by Leiting et al. (2010), who obtained similar results.

Zhang et al. (2011b) developed terpolymers formed by different concentrations of AM, 2-Trimethylammonium Ethyl Methacrylate Chloride (TMAEMC) and 5,5,5Triphenyl-1-Pentene (TP) and found that the rising viscosity of the solutions was highly dependent on the quantity of hydrophobic aromatic groups (TP) in the chains and the polymer concentration. The best viscosification performance was observed above the CAC $\left(0.25 \mathrm{~g} \mathrm{dL}^{-1}\right)$, by favoring intermolecular interactions. Furthermore, they found that the AM-TMAEMC-TP terpolymer had good salinity and temperature resistance.

Lai et al. (2012) conducted a comparative study between PHPA and a terpolymer composed of AM, Acrylic Acid (AA) and N, N-Divinylnonadeca-1,10-Dien-2-Amine (DNDA) and found that the terpolymer had higher salinity and temperature tolerance and excellent shear strength. Besides this, porous medium tests were performed simulating water flooding and polymer flooding with PHPA and HMAP at concentration of $2000 \mathrm{ppm}$ in $\mathrm{NaCl}$ brine at $65{ }^{\circ} \mathrm{C}$. Recovery factors of $59.1 \%, 60.2 \%$ and $63.8 \%$ were observed, respectively. The overall results showed the benefits of polymer flooding to increase oil recovery and the efficiency of the HMAP analyzed as a viscosifying agent for application in EOR.

Leiting et al. (2012) also performed a comparative study between PHPA $3830\left(22 \times 10^{6} \mathrm{~g} / \mathrm{mol}\right)$ and hydrophobically modified polyacrylamide $\left(12 \times 10^{6} \mathrm{~g} / \mathrm{mol}\right)$, commercially known as HAWP 0312, evaluating the viscosities of polymer solutions at $1250 \mathrm{ppm}$ in injection brine and the performance through porous medium tests. They observed that the viscosities of PHPA and HAWP polymer solutions at the shear rate of $7.34 \mathrm{~s}^{-1}$ (an estimated rate at which the fluid passes through the porous medium) were 15.7 and $298 \mathrm{mPa}$ s, respectively. Due to the higher viscosity of the HAWP solution, its $F_{\mathrm{R}}{ }^{*}$ in the porous medium was approximately 3.5 times greater than that of PHPA, leading to a $14.8 \%$ increase in the oil recovery factor. Wang et al. (2012) and Zhang et al. (2012) also conducted comparative studies between commercial PHPA and hydrophobically modified polymers and observed better properties and performance of HMAP under reservoir conditions.

Zou et al. (2013) developed a hydrophobically modified polyacrylamide with cyclodextrin (HCMPAM) and evaluated the stability of this polymer under extreme salinity and temperature conditions compared to a PHPA solution with similar molecular weight. They observed that HCMPAM had higher stability and apparent viscosity in brine with $60000 \mathrm{ppm}$ of Total Dissolved Salts (TDS), with viscosity retention of $45.7 \%$ at $10 \mathrm{~s}^{-1}$ when raising the temperature from $50{ }^{\circ} \mathrm{C}$ to $120^{\circ} \mathrm{C}$, while PHPA presented retention of $8.9 \%$, indicating its lesser stability under severe temperature conditions.

Moreover, Zou et al. (2013) reported that the use of hydrophobically modified polymer increased the oil recovery factor by $5.7 \%$, so they considered it a good alternative for application in reservoirs with high temperature and high mineralization. Deng et al. (2014) also found good salinity resistance of HMAPs and indicated their possible application in reservoirs with high salinity. The same year, Wei and Romero-Zerón (2014) studied the properties of commercial HMAP under reservoir conditions.

According to Zhang et al. (2015), a commercial Hydrophobically Associative Polyacrylamide (HAPAM) with $25.4 \%$ hydrolysis degree can be considered a promising agent for polymer flooding, since it presented higher 
viscosity and superior recovery factor in comparison with a PHPA solution in synthetic brine with 20000 ppm TDS at high temperatures.

Gou et al. (2015) used 3-(2-(2-heptadec-8-enyl-4,5dihydroimidazol-1-yl) ethylcarbamoyl) acrylic acid (NIMA), 3-(diallylamino)-2-hydroxypropyl sulfonate (NDS), AM and AA to produce acrylamide-based copolymers, respectively called AM-AA-NIMA and AM-AANDS-NIMA. They found that the presence of the NDS group provided better properties to the copolymer based on porous simulation tests to compare its efficiency with that of a PHPA solution. The application of the AM-AANDS-NIMA polymer solution at $2000 \mathrm{ppm}$ increased the oil recovery factor by $15.46 \%$ under simulated reservoir conditions.

Jiang et al. (2015) developed a DTHAP copolymer consisting of AM, AA, AMPS and N-Phenethyl-N-Tetradecyl Methacrylamide (PETMAM) and evaluated the influence of temperature, salinity and aging, along with its performance under reservoir conditions. DTHAP showed higher resistance to temperature and salinity and better stability compared to PHPA. The oil recovery factor increased by $9.8 \%$ after injecting $2000 \mathrm{ppm}$ solutions into synthetic brine with $40000 \mathrm{ppm}$ TDS. Thus, the insertion of aromatic groups in acrylamide-based copolymers can be considered promising to improve the performance of polymers in reservoirs with high salinity and temperature.

HMAP injection was evaluated by Patokina (2015) in a project implemented from 2013 to 2015 in the Perm field in Russia, with the injection of a polymeric solution at $1000 \mathrm{ppm}$ under constant monitoring. Process efficiency results were not reported, but they estimated additional oil production of 34.2 million barrels, an increase of $18.93 \%$ in the oil recovery factor.

Zhang et al. (2015) compared the application of PHPA and a Hydrophobically Associative Polyacrylamide (HAPAM) in homogeneous and heterogeneous models, evaluating the possible limitations of the application of associative polymers. They found that the HAPAM $R_{\mathrm{F}}{ }^{*}$ and $R_{\mathrm{RF}}$ tended to be higher than those of PHPA, but the effective viscosity of PHPA was higher than that of HAPAM at 2000 ppm (below the CAC of the hydrophobically modified polymer). At this same concentration, PHPA had better displacement efficiency in homogeneous models, while in heterogeneous models (with a permeability ratio of 2.8) HAPAM performed better. Thus, the application of HAPAM in polymer flooding has some limitations, so further studies should be carried out to improve some properties of this HAPAM.

El-Hoshoudy et al. (2017b) evaluated the influence of concentration, salinity tolerance, and temperature resistance of a HAPAM compared to a PHPA solution. They noted that the optimal concentration of HAPAM was $2000 \mathrm{ppm}$, the polymer exhibited temperature resistance and salinity tolerance, and generally had better properties than PHPA. Furthermore, they were able to recover $20.8 \%$ of the oil by injecting a $2000 \mathrm{ppm}$ solution in brine with $40000 \mathrm{ppm}$ of TDS at temperature of $90^{\circ} \mathrm{C}$, making the formulation a good candidate for application in reservoirs with extreme salinity and temperature conditions. A study of salinity tolerance and temperature resistance of a new HMAP was also conducted by Sarsenbekuly et al. (2017a), with satisfactory results.

Liu et al. (2017a) produced a hydrophobically modified Hydroxyethylcellulose (HEC) with Bromodecane (BD-HMHEC) and evaluated the influence of concentration, salinity, temperature and recovery efficiency compared to water flooding and HEC solution flooding. They observed that the BD-HMHEC solution's CAC value was around $4000 \mathrm{ppm}$ and this solution was more efficient than the HEC solution at high temperatures $\left(60{ }^{\circ} \mathrm{C}\right.$ to $\left.90{ }^{\circ} \mathrm{C}\right)$ and presented greater salinity tolerance. In addition, the use of BD-HMHEC increased oil recovery by $7-14 \%$ compared to HEC injection in simulation tests in porous media.

Wang et al. (2018a) also conducted studies of the application of BD-HMHEC, compared with HEC injection, and evaluated the influence of concentration, porous medium permeability, and injection rate on $R_{\mathrm{F}}{ }^{*}$ and $R_{\mathrm{RF}}$ from simulations of polymer injection in a porous sandstone medium. Initially, they found that $R_{\mathrm{F}}{ }^{*}$ and $R_{\mathrm{RF}}$ were close in the polymeric solutions at $2000 \mathrm{ppm}$, but when reaching the concentration of $4000 \mathrm{ppm}$, BD-HMHEC reached the $\mathrm{CAC}$, which significantly increased the viscosity of the polymeric solution due to the formation of intermolecular interactions, producing higher $R_{\mathrm{F}}{ }^{*}$ and $R_{\mathrm{RF}}$ values. Moreover, they observed that in rock plugs with high and medium permeability and at different injection rates, hydrophobically modified polymer solutions performed better and were considered promising for application in EOR.

The application of BD-HMHEC was also studied by Bai et al. (2018). However, they evaluated the influences of temperature, shear rate and concentration on the viscosity of the polymeric solutions in synthetic brine with $15296 \mathrm{ppm}$ TDS. They reported the formation of hydrophobic aggregates after the CAC, indicating higher viscosifying power of BD-HMHEC, with greater $R_{\mathrm{F}}{ }^{*}$ and $R_{\mathrm{RF}}$ values and oil recovery factors compared to HEC application.

Li et al. (2018) compared the rheological properties of PHPA, HMAP and Cyclodextrin-modified HMAP (HMAP-CD) solutions, to determine the process efficiency by studying the behavior in sandstone-based porous media. They found that the HMAP solution had higher viscosity and elastic modulus $\left(G^{\prime}\right)$ values than the HMAP solution, but the cyclodextrin-modified structure had better properties than the hydrophobically modified polymer. From the porous medium tests, they noted that the PHPA, HMAP and HMAP-CD solutions presented final oil recovery factors equal to $50.0 \%, 68.8 \%$ and $74.5 \%$, respectively. Thus, the incorporation of cyclic groups in the hydrophobically modified structure of polyacrylamides can be considered a promising alternative to improve the efficiency of polymers for EOR. Investigation of modified polyacrylamide with cyclodextrin groups was also performed by Xie et al. (2019).

Azad et al. (2018) conducted a comparative analysis of commercial PHPA and HMAP with molecular weights in 
the range of $16-20 \times 10^{6} \mathrm{~g} / \mathrm{mol}$ and $25 \%$ hydrolysis degree (Flopaam 3630S and Superpusher C319, respectively). They mainly evaluated the effect of hydrophobic associations by comparing the rheological properties of the polymeric solutions at concentrations of 1000 and $2000 \mathrm{ppm}$. They observed a small difference between the viscosities of the solutions at $1000 \mathrm{ppm}$, since the associative polymer showed only intramolecular interactions. A considerable difference was observed between the viscosities of the polymeric solutions at $2000 \mathrm{ppm}$ due to the formation of intermolecular interactions upon reaching the $\mathrm{CAC}$, presenting higher $R_{\mathrm{F}}{ }^{*}$ than the PHPA solution.

Comparative studies between commercial HMAP and PHPA were also carried out by Han et al. (2018), evaluating pore retention and the viscosity of these polymers. They found that the hydrophobically modified structure was the most promising for polymer flooding. Zhong et al. (2018) also compared HMAP and a commercial polymer, evaluating the displacement mechanism of polymer solutions. They concluded that the hydrophobically modified polymer had better properties for application in EOR. Similar studies were also performed by Zhang et al. (2019), who noted that the injection of HMAP in porous media increased the oil recovery factor by $18.7 \%$ compared to PHPA injection.

Abirov et al. (2019) performed porous media tests with commercial PHPA and HMAP solutions simulating conditions of the Turgay Southern Basin reservoir in Kazakhstan, where severe salinity and temperature conditions prevail (92 $000 \mathrm{ppm}$ TDS and $82{ }^{\circ} \mathrm{C}$ ). They reported that the associative polymer was more stable and efficient in the simulated reservoir conditions and recommended its pilot scale application.

Quan et al. (2019) developed a hydrophobically modified xanthan gum (XG-C16) by grafting hexadecyl groups in the hydroxyl groups of the main $\mathrm{XG}$ chain through esterification reactions and compared its properties with those of pure XG. They observed that the solution XG-C16 showed greater temperature resistance and salinity tolerance than the XG solution. They also found that the CAC of XG-C16 was 1600 ppm, and starting at this concentration, there was a significant increase in the viscosity of the solution, indicating the association of the hydrophobic groups of the modified polymer.

Peng et al. (2019) studied the effect of adding silica nanoparticles in hydrophobically modified polyacrylamide with N, N-dimethyl octadecyl ammonium groups (NPHMAP) and found that the presence of silica improved the viscosity and viscoelasticity of the polymeric solution at high temperatures. Thus, the addition of nanofillers was cited as a way to further improve the properties of hydrophobically modified polymers.

In general, hydrophobically modified polymers have better thermal stability, salinity tolerance and oil recovery efficiency than unmodified polymers. Thus, they can be considered promising viscosifying agents for EOR in reservoirs with harsh conditions (high salinity and temperature) (El-Hoshoudy et al., 2017a; Liu et al., 2017a; Zhang et al., 2019). Table 1 shows the summary of the main scientific developments in this area and the evolution of the studies.

\section{Thermo-associative or Thermo-Viscosifying Polymers (TVPs)}

Thermo-Viscosifying Polymers have also emerged as alternatives to improve the efficiency of polymer flooding in reservoirs with high salinity and temperature. TVPs are obtained by chemical modification through the insertion of hydrophobic thermosensitive blocks or grafts having Low Critical Solution Temperature (LCST) in the main chain of a water-soluble polymer. The main thermosensitive groups incorporated in these chains are copolymers of Poly (Ethylene Oxide)-Poly(Propylene Oxide) (PEO-PPO), poly (N-isopropylacrylamide) (PNIPAM) and Macromonomers based on Acrylamide Diacetone (MPAD), among others (Chen et al., 2013; Li et al., 2017; Roy et al., 2013; Sarsenbekuly et al., 2017b; Su and Feng, 2018; Zhu et al., 2013).

These structures behave like water-soluble macromolecules at room temperature, but above the critical association temperature $\left(T_{\text {ass }}\right)$ or LCST, the thermosensitive sequences are reversibly self-aggregated by the formation of physical interactions, forming hydrophobic microdomains that increase the viscosity of the polymeric solution (Kamal and Sultan, 2017; Kamal et al., 2015a; Li et al., 2017; Wang et al., 2010). The schematic representation of the association mechanism of these TVP systems is shown in Figure 3.

Hourdet et al. (1994) developed the first TVP for oil industry application, from the incorporation of $\mathrm{PEO}$ thermosensitive sequences modified with poly(acrylic acid) amine groups. According to the authors, an increase in viscosity was observed at temperatures higher than the LCST and the thermo-viscosification effect was more pronounced in TVP with higher PEO content in its structures, indicating the ability of PEO sequences to form microdomains and increase the viscosity of polymeric solutions.

After this initial study of TVP application in the oil industry, several authors carried out further research in this area (Chen et al., 2013; Leblanc et al., 2015; Li et al., 2017; Reichenbach-Klinke et al., 2018; Sarsenbekuly et al., 2017b; Wang et al., 2010, 2011; Zhu et al., 2013).

Wang et al. (2010) developed a thermo-viscosifying system from free radical copolymerization of Acrylamide (AM) with the macromonomer MPAD, producing the water-soluble copolymer P(AM-co-MPAD). They prepared TVP and PAM solutions in deionized water and brine containing different concentrations of $\mathrm{KCl}$. They then evaluated the influence of temperature on each one. They found that the viscosity of the PAM solution decreased with increasing temperature while the $\mathrm{P}(\mathrm{AM}-\mathrm{co}-\mathrm{MPAD})$ showed the opposite behavior when the TVP reached the $T_{\text {ass }}$. Furthermore, they noted that the $T_{\text {ass }}$ can be adjusted with the variation of the salinity of the system, because the increase in the salt content caused a decrease in the $T_{\text {ass }}$, increasing the viscosity of the polymeric solution at lower temperatures.

Wang et al. (2011) performed a comparative study between $\mathrm{P}$ (AM-co-MPAD) with a molecular weight of $6.5 \times 10^{6} \mathrm{~g} / \mathrm{mol}$ and PHPA with $1.2 \times 10^{7} \mathrm{~g} / \mathrm{mol}$, dissolved 
Table 1. Summary of the main developments regarding hydrophobically modified associative polymers.

\begin{tabular}{lll}
\hline Year & Activity & Authors \\
\hline 1967 & $\begin{array}{l}\text { Developed the first HMAP from the copolymerization of alkyl vinyl } \\
\text { ether with maleic acid }\end{array}$ & Dubin and Strauss \\
1985 & $\begin{array}{l}\text { Patented these systems, highlighting the study of modified } \\
\text { hydroxyethylcellulose }\end{array}$ & Landoll \\
2007 & $\begin{array}{l}\text { Tested HMAPs on a pilot-scale in the Bohai Bay field in China } \\
2009\end{array}$ & $\begin{array}{l}\text { Synthesized a hydrophobically modified acrylamide-based terpolymer } \\
\text { with sodium 2-acrylamide-2-methyl propane sulfonate and 2- }\end{array}$
\end{tabular}
vinylnaphtalene

acrylamide copolymer with N, N-dihexylacrylamide

$2010 \quad$ Compared HMAP and PHPA regarding resistance factor

2011b Developed terpolymers formed by acrylamide, 2-trimethylammonium ethyl methacrylate chloride and 5,5,5-triphenyl-1-pentene

2012 Conducted a comparative study between PHPA and a terpolymer of acrylamide, acrylic acid, and N, N-divinylnonadeca-1,10-dien-2-amine

2012 Performed a comparative study between PHPA and commercial hydrophobically modified polymers

Maia et al.

Leiting et al.

Zhang et al.

Lai et al.

Leiting et al.; Wang et al.; Zhang et al.

Zou et al. cyclodextrin and evaluated their stability under extreme salinity and temperature conditions

2014 Studied the properties of commercial HMAP under reservoir conditions

2015 Considered the commercial HMAP with $25.4 \%$ hydrolysis degree a promising agent for polymer flooding

$2015 \quad$ Used 3-(2-(2-heptadec-8-enyl-4,5-dihydroimidazol-1-yl) ethylcarbamoyl) acrylic acid, 3-(diallylamino)-2-hydroxypropyl sulfonate (NDS), acrylamide and acrylic acid to produce HMAP

Developed a copolymer of AM, AA, AMPS and N-phenethylN-tetradecyl methacrylamide and evaluated the influence of temperature, salinity and aging on its performance

2015 Evaluated the injection of HMAP in the Perm field in Russia

2015 Developed comparative studies between PHPA and HAPAM, evaluating the possible limitations of the application of a hydrophobically associative polymer

$2017 b$

$2017 \mathrm{a}$

$2017 \mathrm{a}$

$2018 \mathrm{a}$
Evaluated the influence of concentration, salinity tolerance, and temperature resistance of HAPAM and PHPA

Studied the salinity tolerance and temperature resistance for a new HMAP

Produced a hydrophobically modified hydroxyethylcellulose with bromodecane and evaluated the influence of several factors
Deng et al.

Wei and Romero-Zéron

Zhang et al.

Gou et al.

Jiang et al.

Patokina et al.

Zhang et al.

El-Hoshoudy et al.

Sarsenbekuly et al.

Liu et al.

Wang et al. 
Table 1. (Continued)

\begin{tabular}{|c|c|c|}
\hline Year & Activity & Authors \\
\hline 2018 & Studied the application of BD-HMHEC & Bai et al. \\
\hline 2018 & $\begin{array}{l}\text { Compared the rheological properties of PHPA, HMAP, } \\
\text { and cyclodextrin-modified HMAP solutions }\end{array}$ & Li et al. \\
\hline 2018 & $\begin{array}{l}\text { Performed comparative studies of commercial HMAP and } \\
\text { PHPA }\end{array}$ & Azad et al.; Han et al.; Zhong et al. \\
\hline 2019 & $\begin{array}{l}\text { Observed that the injection of HMAP in porous media } \\
\text { increased the oil recovery factor by } 18.7 \% \text { compared to } \\
\text { PHPA injection }\end{array}$ & Zhang et al. \\
\hline 2019 & $\begin{array}{l}\text { Conducted studies of modified polyacrylamide with } \\
\text { cyclodextrin }\end{array}$ & Xie et al. \\
\hline 2019 & $\begin{array}{l}\text { Performed porous media testing with commercial PHPA } \\
\text { and HMAP, simulating conditions of the Turgay Southern } \\
\text { Basin reservoir in Kazakhstan }\end{array}$ & Abirov et al. \\
\hline 2019 & $\begin{array}{l}\text { Developed a hydrophobically modified xanthan gum and } \\
\text { compared its properties with unmodified xanthan gum }\end{array}$ & Quan et al. \\
\hline 2019 & $\begin{array}{l}\text { Studied the effect of adding silica nanoparticles in } \\
\text { hydrophobically modified polyacrylamide with } \mathrm{N} \text {, } \\
\text { N-dimethyl octadecyl ammonium groups }\end{array}$ & Peng et al. \\
\hline
\end{tabular}

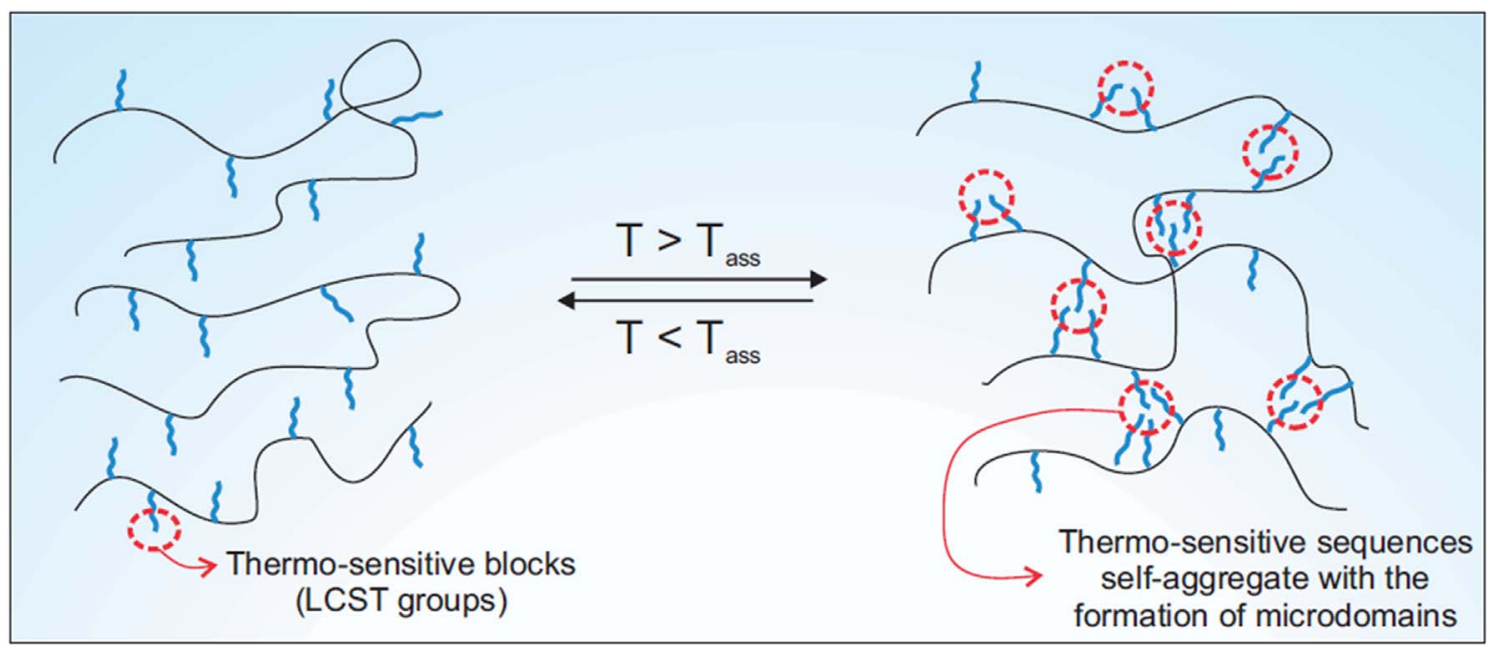

Fig. 3. Association mechanism of thermo-viscosifying polymers.

in synthetic brine with 32868 ppm TDS, simulating the conditions of the Shengli field in China. The effect of polymer concentration was evaluated based on the viscosifying capacity of TVP along with the influence of temperature on the performance of the tested polymers under high salinity conditions.

The PHPA caused a reduction in apparent viscosity and elastic modulus $\left(G^{\prime}\right)$ with increasing temperature, indicating the low stability of this polymer under severe conditions of salinity and temperature. On the other hand,
$\mathrm{P}(\mathrm{AM}-\mathrm{co-MPAD})$ exhibited an increase in viscosity and $G^{\prime}$ reached $T_{\text {ass, }}$, indicating the self-aggregation of thermosensitive sequences and the formation of microdomains. Besides this, the increase in the concentration of the thermosensitive polymer caused a decrease in $\mathrm{T}_{\text {ass }}$ due to the greater number of LCST side chains. According to the results, the studied TVP is a good alternative to decrease the mobility ratio $(M<1)$ of the fluid injected in reservoirs with high salinity and temperature, and consequently to increase the sweep efficiency (Wang et al., 2011). 
Chen et al. (2013) compared solutions containing $2000 \mathrm{ppm}$ of PHPA and P(AM-co-MPAD) in synthetic brine (32 $868 \mathrm{ppm}$ TDS) with molecular weights of $1.2 \times 10^{7} \mathrm{~g} / \mathrm{mol}$ and $5.2 \times 10^{6} \mathrm{~g} / \mathrm{mol}$, respectively. They evaluated the rheological properties of the fluids, the influence of temperature on the behavior of polymeric molecules by cryogenic transmission electron microscopy (Cryo-TEM), and the oil recovery efficiency from tests in a porous medium, simulating conditions of high salinity and temperature of the Shengli Reservoir in China.

They observed loss of viscosity and reduction of $G^{\prime}$ in the PHPA solution, while the TVP solution showed an increase in $G^{\prime}$ and higher viscosity by a factor greater than 7 , with a temperature ranging between $25^{\circ} \mathrm{C}$ and $80{ }^{\circ} \mathrm{C}$. The solutions were tested in a porous medium and oil recovery factors of $2.1 \%$ were observed in the PHPA solution and $13.5 \%$ in the TVP solution, indicating the latter system as promising for EOR. Furthermore, the formation of selfaggregates with increasing temperature was observed by Cryo-TEM analyses, causing an increase in the viscosity of the TVP solution (Chen et al., 2013).

Kamal et al. (2015b) carried out a comparative study of P(AM-AA-MPAD) (a terpolymer with thermo-viscosifying property), PHPA, and a sulfonated tert-butyl acrylamide and acrylamide copolymer P(AM-co-ATBS), with molecular weights of $7.08 \times 10^{6} \mathrm{~g} / \mathrm{mol}, 10 \times 10^{6} \mathrm{~g} / \mathrm{mol}$ and $8 \times 10^{6} \mathrm{~g} / \mathrm{mol}$, respectively. They verified the influence of salinity on the properties of TVP, using deionized water, synthetic seawater with $57643 \mathrm{ppm}$ TDS and formation brine with $213734 \mathrm{ppm}$ TDS. They observed that the thermo-viscosification property was improved with the increase in salinity, since the higher salt concentrations caused a decrease in $T_{\text {ass, }}$, leading to higher viscosity of the solution in formation brine in the evaluated temperature range $\left(20{ }^{\circ} \mathrm{C}\right.$ to $\left.90{ }^{\circ} \mathrm{C}\right)$.

In addition, the authors evaluated the rheological behavior of the three polymers (5000 ppm) studied in synthetic seawater. Initially, there was lower viscosity of TVP compared to the other two commercial polymers, due to its lower molecular weight. With the increase in temperature, the viscosities of the solutions of PHPA and $\mathrm{P}$ (AM-co-ATBS) declined, but increased in the solution of TVP due to the formation of microdomains after $T_{\text {ass. }}$. This behavior is very suitable for polymer injection in EOR, since the low viscosity of the initial solution guarantees the good injectability of the polymer in the reservoir and the high viscosity in severe conditions of salinity and temperature increases the oil recovery (Kamal et al., 2015b).

Leblanc et al. (2015) also compared an AM-ATBS copolymer, commercially known as Flopaam AN132SH, with the commercial thermosensitive polymer DP/TLB132. Like Kamal et al. (2015b), better viscosifying properties of TVP were verified in relation to the commercial copolymer evaluated.

Considering that the injection of polymers is a longterm process, possibly lasting months or years, polymer degradation must be minimized to avoid decreased viscosity of the solution. Therefore, it is very important to evaluate a polymer's stability under reservoir conditions. In this context, Li et al. (2017) studied the thermal stability of a
TVP produced with the macromonomer MPAD $\left(1.01 \times 10^{7} \mathrm{~g} / \mathrm{mol}\right)$ and compared it with the commercial polymer PAM $\left(1.20 \times 10^{7} \mathrm{~g} / \mathrm{mol}\right)$. The solutions with $2000 \mathrm{ppm}$ of polymer in synthetic brine with $101000 \mathrm{ppm}$ TDS were evaluated at temperatures of $45^{\circ} \mathrm{C}$ and $85{ }^{\circ} \mathrm{C}$.

Viscosity losses of $4 \%$ and $52.5 \%$ were observed in the solutions of PHPA after 30 days of aging at $45{ }^{\circ} \mathrm{C}$ and $85{ }^{\circ} \mathrm{C}$, respectively. For TVP solutions, losses of $18.7 \%$ and $74.3 \%$ were observed at $45{ }^{\circ} \mathrm{C}$ and $85^{\circ} \mathrm{C}$, respectively. Thus, the TVP solution showed more pronounced degradation in reservoir conditions, and thus had a higher percentage of viscosity loss. Furthermore, tests were carried out in a porous medium simulating temperature conditions in the Daqing $\left(45^{\circ} \mathrm{C}\right)$ and Shengli $\left(85^{\circ} \mathrm{C}\right)$ fields in China. The TVP system showed good injectability and a higher $R_{\mathrm{F}}{ }^{*}$, contributing to oil recovery factors of $16.4 \%$ and $15.5 \%$ at $45^{\circ} \mathrm{C}$ and $85^{\circ} \mathrm{C}$, respectively, while PAM had oil recovery factors of $12.0 \%$ and $9.2 \%$. Therefore, this TVP system was considered a promising option for application as a viscosifying agent in EOR fluids (Li et al., 2017).

Akbari et al. $(2017 \mathrm{a}, \mathrm{b})$ evaluated the performance of four acrylamide-based copolymers containing 2-acrylamide-2-methylpropane sulfonate groups (AMPS), one of them with a thermosensitive side group. The aim of this study was to evaluate the effects of salinity, injection water hardness, shear rate and temperature on the viscosity of polymeric solutions. The authors found that the thermosensitive polymer had better properties and was a promising viscosifying agent for application in EOR.

Sarsenbekuly et al. (2017b) observed that the viscosifying behavior of commercial TVPs was strongly dependent on polymer concentration, salinity and temperature. When comparing a PHPA $\left(16 \times 10^{6} \mathrm{~g} / \mathrm{mol}\right)$ and TVP $\left(6 \times 10^{6} \mathrm{~g} / \mathrm{mol}\right)$ in synthetic brine with $9583.74 \mathrm{ppm}$ TDS at temperature of $30^{\circ} \mathrm{C}$, they observed higher viscosity and elastic modulus of TVP in comparison with PHPA, despite the latter's lower molecular weight. In addition, they noted that the temperature was paramount in determining the properties of this polymer, since its viscosity increased six-fold by raising the temperature from $30{ }^{\circ} \mathrm{C}$ to $90{ }^{\circ} \mathrm{C}$. Similar results were observed by ReichenbachKlinke et al. (2018).

Wang et al. (2018b) performed a comparative study between the copolymer of AM, NaAA and AA (PAMA) and three TVPs formed by PAMA and Pluronic $\left(\mathrm{PEO}_{\mathrm{n}}-\right.$ $\mathrm{PPO}_{\mathrm{m}}-\mathrm{PEO}_{\mathrm{n}}$ triblock copolymer): TVP-P1 (PAMA and $\mathrm{PEO}_{100}-\mathrm{PPO}_{65}-\mathrm{PEO}_{100}$ ); TVP-P2 (PAMA and $\mathrm{PEO}_{132}$ $-\mathrm{PPO}_{50}-\mathrm{PEO}_{132}$ ); and TVP-P3 (PAMA and $\mathrm{PEO}_{76}-$ $\left.\mathrm{PPO}_{29}-\mathrm{PEO}_{76}\right)$. Initially, the rheological behavior of the TVP solutions was evaluated and a higher viscosity of the TVP-P1 solution was found in the temperature analysis range $\left(25{ }^{\circ} \mathrm{C}\right.$ to $\left.95{ }^{\circ} \mathrm{C}\right)$, due to the presence of more PPO groups, responsible for forming physical connections above the $T_{\text {ass. }}$. The behavior of TVPs was highly dependent on their chemical structures and the content of thermosensitive group in the polymer chain.

TVP-P1 was evaluated regarding thermal stability and by tests in a porous medium to check the injectivity and the oil recovery potential in comparison with the PAMA 
Table 2. Summary of the main activities involving TVPs.

\begin{tabular}{|c|c|c|}
\hline Year & Activity & Authors \\
\hline 1994 & $\begin{array}{l}\text { Developed the first polymer with thermo-viscosifying property for the } \\
\text { oil industry }\end{array}$ & Hourdet et al. \\
\hline 2010 & $\begin{array}{l}\text { Produced a thermo-viscosifying system from AM and MPAD } \\
\text { copolymerization }\end{array}$ & Wang et al. \\
\hline 2011 & $\begin{array}{l}\text { Conducted a comparative study between } \mathrm{P}(\mathrm{AM}-\mathrm{co}-\mathrm{MPAD}) \text { and } \\
\text { PHPA, evaluating the effects of several factors }\end{array}$ & Wang et al. \\
\hline 2013 & $\begin{array}{l}\text { Developed poly [acrylamide-poly (oxyethylene octylphenol ether acryl } \\
\text { ester)] and evaluated its thermo-associative capacity }\end{array}$ & Zhu et al. \\
\hline $2015 b$ & $\begin{array}{l}\text { Carried out a comparative study between the terpolymer with thermo- } \\
\text { viscosifying property P(AM-AA-MPAD), PHPA and P(AM-co- } \\
\text { ATBS) }\end{array}$ & Kamal et al. \\
\hline 2015 & $\begin{array}{l}\text { Evaluated the performance of AM-ATBS copolymer and commercial } \\
\text { TVP }\end{array}$ & Leblanc et al. \\
\hline 2017 & $\begin{array}{l}\text { Studied the thermal stability of TVP produced with the } \\
\text { macromonomer MPAD }\end{array}$ & Li et al. \\
\hline $2017 \mathrm{a}$ and $2017 \mathrm{~b}$ & $\begin{array}{l}\text { Evaluated the performance of four acrylamide-based copolymers } \\
\text { containing } 2 \text {-acrylamide-2-methylpropane sulfonate groups and one } \\
\text { them with thermosensitive side groups }\end{array}$ & Akbari et al. \\
\hline $2017 \mathrm{~b}$ & $\begin{array}{l}\text { Observed that the viscosifying behavior of commercial TVPs is } \\
\text { extremely dependent on polymer concentration, salinity and } \\
\text { temperature }\end{array}$ & Sarsenbekuly et al. \\
\hline 2018 & Performed studies with commercial TVPs & Reichenbach-Klinke et al. \\
\hline $2018 b$ & $\begin{array}{l}\text { Performed a comparative study between the copolymer of AM, NaAA, } \\
\text { and AA (PAMA) and the TVPs formed by PAMA and Pluronic } \\
\left(\mathrm{PEO}_{\mathrm{n}}-\mathrm{PPO}_{\mathrm{m}}-\mathrm{PEO}_{\mathrm{n}}\right)\end{array}$ & Wang et al. \\
\hline
\end{tabular}

solution. There was a $10.3 \%$ loss in the viscosity of the TVP solution and $26.2 \%$ for PAMA, indicating greater resistance of this TVP to thermal degradation. Furthermore, the thermo-viscosifying system showed good injectability and tests simulating conditions in the Daqing reservoir indicated an increase of $2.1 \%$ in the oil recovery factor with the application of the thermosensitive system (Wang et al., 2018b).

In general, TVP systems have good properties and can be considered promising options for use in harsh reservoir conditions (high salinity and temperature) (Divers et al., 2018; Kamal et al., 2015b; Li et al., 2017; Roy et al., 2013; Su and Feng, 2018; Wang et al., 2011). The summary of the main activities investigated regarding TVPs and the evolution of studies is shown in Table 2 .

\section{Polymer systems with nanoparticles}

The application of nanoparticles in polymer flooding is being investigated as an alternative to improve the rheological properties, salinity and temperature tolerance and shear resistance of injection polymers, and hence to increase the oil recovery factor. Nanoparticles are materials with particle sizes in the range of 1-100 nm, and their performance is directly related to their small size, which allows a larger contact surface, resulting in nanofluids with satisfactory properties for application in enhanced oil recovery (Cheraghian and Hendraningrat, 2016; Gbadamosi et al., 2019a; Rellegadla et al., 2018).

Several research groups have studied the formation of hybrid systems of nanofillers with polymers for EOR application, such as the use of nanoparticles of silica $\left(\mathrm{SiO}_{2}\right)$, titanium oxide $\left(\mathrm{TiO}_{2}\right)$, aluminum oxide $\left(\mathrm{Al}_{2} \mathrm{O}_{3}\right)$, iron oxide $\left(\mathrm{Fe}_{2} \mathrm{O}_{3}\right)$ and nickel oxide $(\mathrm{NiO})$, as well as nanoclays and carbon nanotubes, among others (Abdullahi et al., 2019; Cheraghian, 2016; Corredor et al., 2019; Giraldo et al., 2017; Haruna et al., 2019; Nguyen et al., 2015; Rellegadla et al., 2018; Zeyghami et al., 2014; Zhu et al., 2014a).

Zhu et al. (2014a) carried out a comparative study between a Hydrophobically Associating Polyacrylamide (HAPAM) and a hybrid system of HAPAM/silica nanoparticles and evaluated the rheological behavior of aqueous solutions. They found that the addition of nanosilica 


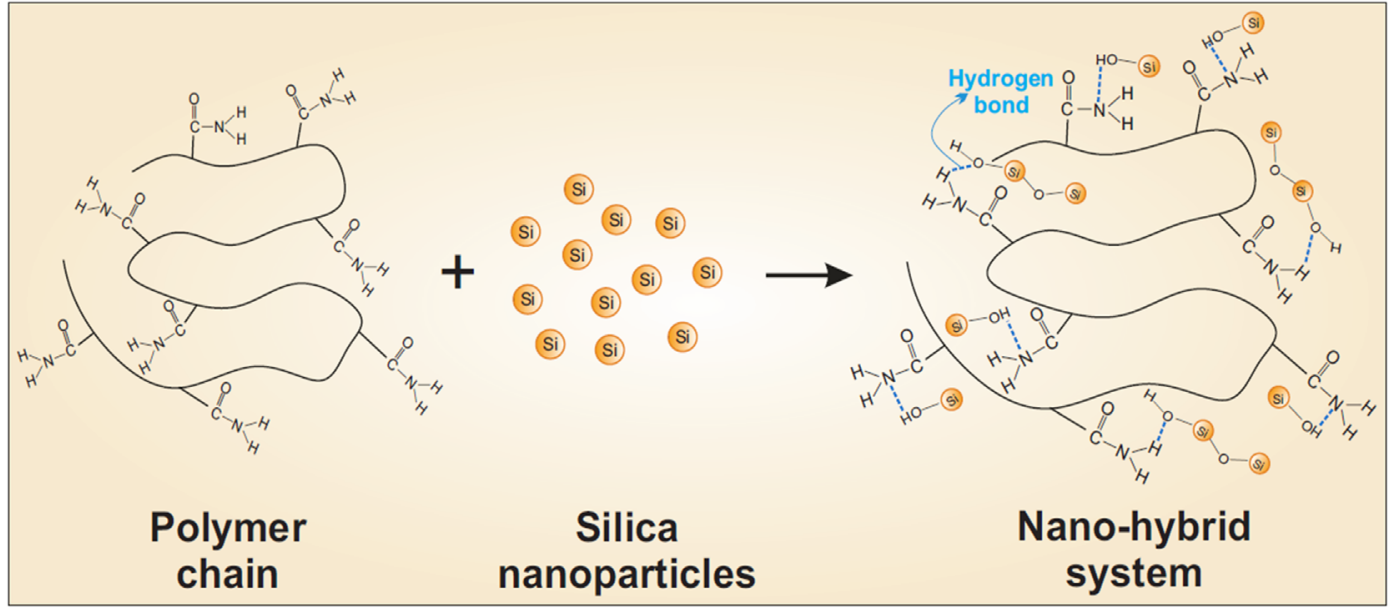

Fig. 4. Representative scheme of the formation of a nano-hybrid system.

promoted an increase in the apparent viscosity and storage modulus of the HAPAM solutions, and the viscosity increased considerably by raising the concentration of silica nanoparticles from $0.5 \mathrm{wt} \%$ to $2.5 \mathrm{wt} \%$. Hybrid systems also showed greater thermal stability and salt tolerance, the latter effect due to the interaction between the nanosilica and the polymer through hydrogen bonding between the silanol groups of the nanosilica and amide groups of HAPAM, reinforcing the polymeric structure (Fig. 4).

In the same year, Zhu et al. (2014a) studied the effect of silica nanoparticles on the properties of solutions of Hydrophobically Associating Partially Hydrolyzed Polyacrylamide (HAPHPA) and evaluated the efficiency of the nano-hybrid system in increasing the oil recovery factor under high salinity and temperature conditions. Solutions of $5000 \mathrm{ppm}$ HAPHPA were prepared in brine with 32868 ppm TDS $\left(\left[\mathrm{Ca}^{2+}\right]+\left[\mathrm{Mg}^{2+}\right]=873 \mathrm{ppm}\right)$. The addition of $1.0 \mathrm{wt} \%$ silica nanoparticles increased the viscosity from $26.3 \mathrm{mPa} \mathrm{s}$ to $136.9 \mathrm{mPa} \mathrm{s}$, under shear rate of $10 \mathrm{~s}^{-1}$ at $85{ }^{\circ} \mathrm{C}$. Furthermore, core flooding tests showed that HAPHPA injection caused an oil recovery factor of $5.44 \%$, while for HAPHPA-nanosilica hybrid the factor was $10.57 \%$. Thus, this system can be considered promising for EOR application.

Cheraghian (2016) evaluated the effect of incorporating titanium dioxide nanoparticles on the viscosity of PHPA solutions and compared the oil recovery potential of PHPA solutions and the PHPA $/ \mathrm{TiO}_{2}$ hybrid nanosystems. For this, solutions of $3150 \mathrm{ppm}$ of PHPA in synthetic brine were used and $\mathrm{TiO}_{2}$ nanoparticles were added at concentrations of $1.9,2.1,2.3$, and $2.5 \mathrm{wt} \%$. The systems were evaluated for viscosity and in core flooding tests. The author observed that the addition of $\mathrm{TiO}_{2}$ promoted an increase in the viscosity of PHPA solutions (shear rate of $10 \mathrm{~s}^{-1}$ ), and the system with $2.3 \mathrm{wt} \%$ of $\mathrm{TiO}_{2}$ showed the best results. Furthermore, the application of this nano-hybrid system increased oil recovery by $4 \%$ compared to the application of the PHPA solution. Thus, the use of these nanoparticles was considered a promising method to improve the performance of this polymer for EOR.
Rezaei et al. (2016) carried out a comparative study of the rheological properties of PHPA solutions and systems formed by the addition of Surface Modified Clay Nanoparticles (SMCN) with tetraethylammonium chloride (PHPA$\mathrm{SMCN})$. The viscosities of the solutions were evaluated at temperatures of 30,60 and $90{ }^{\circ} \mathrm{C}$ and the salinity tolerance of the polymer systems was verified by varying the salinity of the brine from 2000 to 100000 ppm TDS $(76.2 \% \mathrm{NaCl}$, $1.5 \% \mathrm{MgCl}_{2}, 3.7 \% \mathrm{CaCl}_{2}$, and $\left.18.6 \% \mathrm{Na}_{2} \mathrm{SO}_{4}\right)$. They observed that the addition of SMCN promoted an increase in the viscosity of the solutions, improving the resistance to salinity and temperature and increasing stability. In addition, tests in porous media were performed and indicated that the application of SMCN can be promising for polymer flooding, because it increased the oil recovery factor by $33 \%$ compared to the PHPA application.

Giraldo et al. (2017) evaluated the effect of applying $\mathrm{SiO}_{2}$ nanoparticles on decreasing thermal degradation and improving the rheological behavior of PHPA. Stability tests were carried out under an oxidative atmosphere and without the presence of oxygen $\left(70{ }^{\circ} \mathrm{C}\right.$ for 14 days $)$, which indicated greater thermal stability of hybrid systems formed with silica nanoparticles, with a viscosity reduction of up to $46 \%$ in relation to the PHPA solutions. In addition, the application of these nanoparticles increased the viscosity of the polymeric solutions, and thus would allow the application of a lower dosage of PHPA in the polymer flooding process, reducing operating costs.

Zheng et al. (2017) studied the incorporation of silica nanoparticles (DNS) and $\mathrm{SiO}_{2}$ with surface modified by the silane coupling agents hexadecyltrimethoxysilane (DNS-HD) and hexamethyldisilazane (DNS-HM) in aqueous PHPA solutions and evaluated the application of nanosystems for EOR under simulated conditions of the Shengli field in China. Rheological analyses of the solutions in Shengli brine, at temperatures of $30-80{ }^{\circ} \mathrm{C}$, indicated that the addition of DNS increased the viscosity of the PHPA solutions, and this effect was stronger at higher $\mathrm{SiO}_{2}$ concentrations. The best result was obtained with $2.0 \mathrm{wt} \%$ DNS. However, nanosilica surface-modification 
further improved the apparent viscosity and thermal stability of the hybrid systems. This occurred because the modifications provided a slightly hydrophobic character to the nanoparticles, which tended to associate and form microdomains in the aqueous solution tested. Therefore, the systems formed by this nanofiller had hydrogen bonds characteristic of silanol groups with PHPA, which together with the associative effect of the hydrophobic groups improved the properties of the PHPA solutions.

In addition, displacement tests in core flooding systems at $80{ }^{\circ} \mathrm{C}$ were performed, which indicated oil recovery factors of $7.08 \%$ for PHPA, 7.70\% for PHPA-DNS, $9.25 \%$ for PHPA-DNS-HD and $10.54 \%$ for PHPA-DNS-HM. Therefore, the modifications were favorable to improve the oil recovery factor and the DNS-HM nanoparticles were considered promising to improve the polymer flooding process (Zheng et al., 2017).

Liu et al. (2017b) investigated the association of nanofiller with Hydrophobically Modified Polyacrylamide (HAPAM) by inserting silica nanoparticles in the polymeric matrix (SHAPAM). Polymer solutions containing 2000 ppm of PHPA, HAPAM, and SHAPAM were compared for application in reservoirs with severe salinity and temperature conditions. HAPAM showed better properties than PHPA, but the insertion of nanoparticles (SHAPAM) caused the formation of more rigid structures, with viscosity values 3.6 times higher than the HAPAM solution, along with high tolerance to salinity and temperature, indicating the insertion of nanoparticles as a promising method. In addition, the oil recovery factors with the injection of PHPA, HAPAM and SHAPAM solutions were $9.2 \%$, $12.5 \%$ and $20 \%$, respectively.

$\mathrm{Hu}$ et al. (2017) also evaluated the incorporation of $\mathrm{SiO}_{2}$ nanoparticles in PHPA solutions, under high temperature and salinity conditions, and found an improvement in the rheological properties of PHPA solutions by transforming them into PHPA-SiO 2 hybrid nanosystems.

Rellegadla et al. (2018) studied the effect of incorporating nickel Nanoparticles (NPs) in Xanthan Gum (XG) solutions and evaluated the efficiency of the nanopolymer system for the recovery of residual oil from sand packed bioreactors with oil collected from the Ankleshwar oil fields in India, in comparison with the injection of a solution of XG and suspension of nickel nanoparticles. They found that the insertion of nickel nanoparticles increased the intrinsic viscosity of the $\mathrm{XG}$ solution, and the recovery tests with the XG solution, NPs and XG-NPS system indicated oil recoveries of $4.48 \%, 4.58 \%$, and $5.98 \%$, respectively. Hence, they considered the use of nanopolymer systems to be a good alternative to improve the polymer flooding process.

Corredor et al. (2019) also analyzed the incorporation of nanoparticles in xanthan gum solutions. They observed that the addition of surface-modified $\mathrm{SiO}_{2}$ and unmodified $\mathrm{SiO}_{2}$ favored the increase in the viscosity of XG solutions, while $\mathrm{TiO}_{2}, \mathrm{Al}_{2} \mathrm{O}_{3}, \mathrm{Fe}(\mathrm{OH})_{3}$ nanoparticles did not promote increased viscosity.

Haruna et al. (2019) evaluated the effect of the incorporation of Multi-Walled Carbon Nanotubes (MWCNTs) on the rheological properties and efficiency of oil recovery using solutions of copolymers and acrylamide terpolymers, aiming at application in reservoirs with high salinity and temperature. They noted that the incorporation of MWCNTs improved the viscoelasticity and viscosity of the polymeric dispersions. In addition, MWCNTs increased the oil recovery efficiency at $85{ }^{\circ} \mathrm{C}$ in American Petroleum Institute $(A P I)$ brine and in alkaline $\mathrm{pH}$ brine. The results demonstrated this is an alternative to improve the performance of polymers applied in EOR.

Abdullahi et al. (2019) evaluated the incorporation of $\mathrm{Al}_{2} \mathrm{O}_{3}, \mathrm{SiO}_{2}$, and $\mathrm{TiO}_{2}$ nanoparticles in PHPA solutions in different brines at varying temperatures. They found that the addition of $0.1 \mathrm{wt} \%$ of nanoparticles increased the salt tolerance of PHPA solutions, since the nanoparticles have a shielding effect against the cations present in synthetic brines containing divalent cations. Thus, the hybrid nanosystems had better rheological performance than the pure PHPA solution, and the PHPA $/ \mathrm{Al}_{2} \mathrm{O}_{3}$ system had the highest viscosity, followed by PHPA $/ \mathrm{SiO}_{2}$ and $\mathrm{PHPA} / \mathrm{TiO}_{2}$, with the lowest viscosity among the hybrid nanosystems. In addition, oil recovery tests were carried out in packed sand saturated with intermediate oil, where PHPA $/ \mathrm{Al}_{2} \mathrm{O}_{3}$, $\mathrm{PHPA} / \mathrm{SiO}_{2}$, and $\mathrm{PHPA} / \mathrm{TiO}_{2}$ systems produced additional recoveries of $15 \%, 10 \%$, and $6 \%$, respectively, in relation to the solution of PHPA. Thus, the use of nanoparticles can be considered promising to improve the polymer flooding process.

Yadav et al. (2020) studied the influence of salinity, temperature, shear rate, nanofiller concentration and polymer concentration on the rheological behavior of PHPA/ $\mathrm{SiO}_{2}$ hybrid nanosystems. They observed that the increase in polymeric concentration and content of silica nanoparticles favorably acted by increasing the viscosity of the systems. The salinity and shear rate had a negative effect on rheological behavior, reducing the viscosities of the systems. The evaluation of the influence of temperature (80.15 to $110.68{ }^{\circ} \mathrm{C}$ ) showed that the viscosity decreased with rising temperature until $107^{\circ} \mathrm{C}$, after which the viscosity increased due to the interaction of long chains with the nanoparticles used in high concentration, increasing the cohesive force in the solution and the viscosity. Thus, the addition of a high concentration of silica nanoparticles can be a good option for reservoirs with high temperatures.

Haruna et al. (2020) studied the effects of the incorporation of nanoparticles on the rheological properties, thermal stability and degradation of PAM solutions under HTHS conditions. For this, silica nanoparticles and $\mathrm{SiO}_{2}$ nanoparticles modified with (3-aminopropyl) triethoxysilane $\left(\mathrm{MSiO}_{2}\right)$ were used, forming the hybrid nanosystems $\mathrm{SiO}_{2} / \mathrm{PAM}$ and $\mathrm{MSiO}_{2} / \mathrm{PAM}$, respectively. They found that the addition of nanoparticles increased the viscosity, thermal stability and salinity tolerance of the polymer systems. The PAM, $\mathrm{SiO}_{2} / \mathrm{PAM}$, and $\mathrm{MSiO}_{2} / \mathrm{PAM}$ solutions showed viscosity reductions of $75 \%, 45 \%$, and $10 \%$, respectively, after aging for 90 days. This indicates that the modification of the surface of the nanoparticles can further improve the properties of hybrid nanopolymer systems. Furthermore, enhanced oil recovery tests were carried out simulating HTHS conditions at $85^{\circ} \mathrm{C}$ and with solutions in API brine and formation brine of the Ghawar field in Saudi Arabia. The addition of nanoparticles 
Table 3. Summary of the main activities carried out involving polymer systems with nanoparticles.

\begin{tabular}{lll}
\hline Year & Activity & Authors \\
\hline $2014 \mathrm{a}$ & $\begin{array}{l}\text { Compared the rheological behavior between a Hydrophobically Associating } \\
\text { Polyacrylamide (HAPAM) and a hybrid system of HAPAM/silica } \\
\text { nanoparticles in aqueous solutions }\end{array}$ & Zhu et al
\end{tabular}
nanoparticles in aqueous solutions

Zhu et al. Hydrophobically Associating Partially Hydrolyzed Polyacrylamide (HAPHPA) viscosity of PHPA solutions and compared the oil recovery potential of PHPA solutions and the $\mathrm{PHPA} / \mathrm{TiO}_{2}$ hybrid nanosystems solutions and systems formed by the addition of surface-modified clay nanoparticles

2017 Evaluated the effect of applying $\mathrm{SiO}_{2}$ nanoparticles on decreasing thermal degradation and rheological behavior of PHPA

Rezaei et al.

Conducted studies of the association of silica nanoparticles with hydrophobically modified polyacrylamide

2018 Studied the effect of incorporating nickel Nanoparticles (NPs) in Xanthan Gum (XG) solutions

2019 Conducted studies of the incorporation of nanoparticles $\left(\mathrm{SiO}_{2}, \mathrm{TiO}_{2}, \mathrm{Al}_{2} \mathrm{O}_{3}, \mathrm{Fe}\right.$ $(\mathrm{OH})_{3}$ ) in Xanthan Gum (XG) solutions

2019 Evaluated the effect of the incorporation of Multi-Walled Carbon Nanotubes (MWCNTs) on the rheological properties and efficiency of oil recovery using solutions of copolymers and acrylamide terpolymers

Giraldo et al.

Zheng et al.

Liu et al.

$\mathrm{Hu}$ et al.

Rellegadla et al.

Corredor et al.

Haruna et al.

Abdullahi et al.

Developed a study of the influence of salinity, temperature, shear rate, nanofiller concentration, and polymer concentration on the rheological behavior of PHPA $/ \mathrm{SiO}_{2}$ hybrid nanosystems modified with (3-aminopropyl) triethoxysilane $\left(\mathrm{MSiO}_{2}\right)$ on the rheological properties, thermal stability and degradation of PAM solutions

increased the oil recovery factor in both brines and the best system was $\mathrm{MSiO}_{2} / \mathrm{PAM}$. So, the addition of these modified nanoparticles can improve oil recovery under harsh conditions.

In general, the application of nanoparticles for the production of hybrid nanopolymer systems improves the performance of the injection polymers applied in EOR, increasing the viscosity of the systems and improving oil recovery (Abdullahi et al., 2019; Cheraghian, 2016; Liu et al., 2017b; Rezaei et al., 2016; Zheng et al., 2017; Zhu et al., 2014b). The summary of the main activities carried out involving application of nanoparticles and the evolution of studies is shown in Table 3.

The summary of the main mechanisms of action and advantages of the viscosifying polymer systems to improve the polymer flooding process can be observed in Table 4 . 
Table 4. Summary of the main mechanisms of action and advantages of viscosifying polymer systems to improve the polymer flooding process.

\begin{tabular}{|c|c|c|}
\hline System & Mechanism & Advantages ${ }^{*}$ \\
\hline $\begin{array}{l}\text { Hydrophobically Modified } \\
\text { Associative Polymers } \\
\text { (HMAP) }\end{array}$ & $\begin{array}{l}\text { Hydrophobic association effect of } \\
\text { the groups inserted in the main chain }\end{array}$ & $\begin{array}{l}\text { - Better thermal stability and tolerance to } \\
\text { salinity; } \\
\text { - Substantial increase in viscosity above the } \\
\text { CAC; } \\
\text { - Increased oil recovery factor. }\end{array}$ \\
\hline $\begin{array}{l}\text { Thermo-Viscosifying } \\
\text { Polymers (TVP) }\end{array}$ & $\begin{array}{l}\text { Thermosensitive sequences are } \\
\text { reversibly self-aggregating by the } \\
\text { formation of physical interactions, } \\
\text { forming hydrophobic microdomains }\end{array}$ & $\begin{array}{l}\text { - Improved the polymer performance } \\
\text { under HTHS conditions; } \\
\text { - Increased viscosity with higher temperatures, } \\
\text { above LCST; } \\
\text { - Increased oil recovery factor. }\end{array}$ \\
\hline $\begin{array}{l}\text { Polymer systems with } \\
\text { nanoparticles }\end{array}$ & $\begin{array}{l}\text { Physical or chemical interactions } \\
\text { between nanoparticles and functional } \\
\text { groups in the polymer chain }\end{array}$ & $\begin{array}{l}\text { - Improved thermal stability and salinity } \\
\text { tolerance; } \\
\text { - Improved rheological properties; } \\
\text { - Allowed application of lower polymer } \\
\text { concentration; } \\
\text { - Increased oil recovery factor. }\end{array}$ \\
\hline
\end{tabular}

${ }^{*}$ Advantages in relation to the application of PHPA solutions for polymer flooding.

\section{Artificial intelligence application}

Over the last decades, the use of genetic programming and artificial intelligence has gained great importance in the reservoir engineering, as it allows the creation of computer models to obtain satisfactory results with high precision in several oil fields. These devices were created to generate models and accurate correlations from a database with different field conditions, aiming to determine the best working conditions to obtain promising results, based on the genetic algorithm that consists of a metaheuristic technique of search belonging to evolutionary computing algorithms group applied to optimize solutions and detect problems (Amirian et al., 2018; Kamari et al., 2017; Rostami et al., 2016, 2017c).

Considering that performing some tests on a laboratory scale takes time, extensive planning, and relatively high costs, the mathematical strategy of genetic programming emerges as an alternative to estimate results from a wide range of experimental conditions acquired from databases, allowing optimization of results. Thus, several authors have studied the application of genetic programming in many areas of science and engineering, obtaining promising results over the last decade (Kamari et al., 2017; KarkevandiTalkhooncheh et al., 2018; Rostami et al., 2017c, 2018a).

Artificial Neural Networks (ANNs) correspond to information processing methods capable of creating models from complex data input and output relationships, identifying important data patterns, and being widely applied to process control, analysis techniques, data processing, experimental value estimation, failure detection, pattern recognition, among others (Amirian et al., 2018; Corredor-Rojas et al., 2018; Rostami et al., 2016, 2018a).

Artificial neural networks can also be used to develop models that allow estimating the carbon dioxide $\left(\mathrm{CO}_{2}\right)$ adsorption equilibrium in activated carbon. Normally, this equilibrium is determined by Sips and Langmuir isotherms, however, the development of algorithms based on artificial neural networks can be an alternative for the acquisition of more efficient results and without the restriction of work at constant temperatures (Rostami et al., 2018a). Thus, Rostami et al. (2018a) developed a multi-layer feed-forward neural network algorithm to estimate $\mathrm{CO}_{2}$ adsorption from a wide range of thermodynamic conditions found in the literature. It was verified that the developed model showed greater precision, performance and presents itself as a more promising method when compared to traditional isotherms.

To estimate the efficiency of the thermal enhanced oil recovery process, Rostami et al. (2016) developed an innovative Least Square Support Vector Machine (LS-SVM) algorithm to create a model capable of predicting the effective thermal conductivity in dry sandstones. This method consists of an artificial intelligence technique that is more advantageous than traditional techniques based on artificial neural networks because they present high reproducibility, accuracy, and greater reliability of the results. Thus, 280 experimental data of effective thermal conductivity in dry sandstones present in the literature were collected, containing information on temperature, pressure, porosity, and solid density. Then, the LS-SVM model was developed to predict the effective thermal conductivity. It was found that the method is more accurate than traditional artificial intelligence models, allowing for satisfactory and reproducible results to improve the thermal enhanced oil recovery and the traditional numerical simulation in dry sandstones.

In addition to artificial intelligence methods known as ANN and SVM (computational models that establish complex relationships and do not have an obvious correlation between input and output conditions), geneticbased calculations such as Genetic Programming (GP), 
Gene Expression Programming (GEP), and Genetic Algorithm (GA) can be used to obtain greater accuracy of correlations, more practical and simpler models for users, and can be integrated with conventional software and simulators to determine accurate correlations for each step of a study process (Rostami et al., 2017a, b).

Rostami et al. (2017c) used the GP method to estimate the interfacial tension between hydrocarbon/water in reservoirs, starting from the association of mathematical tools and a wide range of experimental conditions obtained from literature data. Thus, the use of artificial intelligence allowed to obtain a fast and efficient correlation system to estimate interfacial tension values, making it possible to obtain satisfactory results for the oil industries. GP was also used as a mathematical strategy by Rostami et al. (2017a) to estimate the thermal conductivity of supercritical $\mathrm{CO}_{2}$. Thus, it was observed that the developed algorithm presents a good correlation and can be promising to estimate the thermal conductivity in industrial processes that use supercritical $\mathrm{CO}_{2}$.

The GEP method was used to estimate the solubility of $\mathrm{CO}_{2}$ in processes of $\mathrm{CO}_{2}$ flooding in oil reservoirs, aiming to improve this method of enhanced oil recovery. For this, the GEP soft computing technique was developed to establish correlations of the solubility of $\mathrm{CO}_{2}$ in dead and live oil systems. Furthermore, they evaluated the predictive ability of ANN for the solubility of $\mathrm{CO}_{2}$ (using comprehensive data from literature databases) and compared it with the GEP model. Thus, it was found that the ANN technique presented slightly better results only in simulations with live crude oil, but in general, the GEP method is a reliable and highly accurate model to estimate the solubility of $\mathrm{CO}_{2}$ in live and dead oils, being a promising algorithm for this application (Rostami et al., 2017b).

Kamari et al. (2017) developed a comparative study on the estimates of interfacial tension between $\mathrm{CO}_{2}$-brine from the GEP, LS-SVM, and Decision Tree (DT) modeling methods with the ANN data found in the open literature. Thus, it was found that the DT and ANN methods were the most accurate models to determine the interfacial tension between $\mathrm{CO}_{2}$-brine. The DT consists of a regression decision tree modeling, where the dataset is subdivided into smaller groups (a tree with nodes that represent the subsets), after building the tree with the data, cross-validation and the estimate is obtained through the model. In addition, it was found that the GEP technique had the lowest reliability and precision for determining the interfacial tension between $\mathrm{CO}_{2}$-brine, however, it is the model that can provide faster estimates for the process.

In addition to the promising applications of artificial intelligence and genetic programming in estimating important parameters for reservoir engineering and process improvement, these methods can also be applied to estimate and optimize polymer flooding processes in enhanced oil recovery, through estimating values of viscosity of polymeric solutions and the prediction of the performance of these injection fluids in reservoirs. In this context, several authors have developed researches focused on this area (Rezaian et al., 2010; Kang et al., 2016; Amirian et al., 2018; Corredor-Rojas et al., 2018; Rostami et al., 2018b).
Rezaian et al. (2010) applied the artificial neural network model to estimate the minimum amount of Polyvinyl Acetate (PVA) needed to increase water viscosity and simultaneously promote a decrease in oil viscosity, and thus improve the polymer flooding process. To test the effectiveness of the created network, experimental tests were carried out and these showed agreement between the estimated percentages and those obtained in the experiment. Thus, it was found that the ideal amount of PVA for this application was $15.0 \%$ and to recover $100 \mathrm{bbl}$ of oil it would be necessary to use about $7.5 \mathrm{bbl}$ of polymer. Thus, it appears that the use of artificial intelligence can be a promising alternative to improve EOR processes.

Kang et al. (2016) used artificial neural network models to estimate the viscosity of polymer solutions of three commercial acrylamide-based polymers, known as Flopaam 3330S, Flopaam 3630S and AN125, with hydrolysis degrees between $25 \%$ and $30 \%$ and molecular weights of $8.0 \times 10^{6} \mathrm{~g} / \mathrm{mol}, 20.0 \times 10^{6} \mathrm{~g} / \mathrm{mol}$ and $8.0 \times 10^{6} \mathrm{~g} / \mathrm{mol}$, respectively. For this, parameters that influence the viscosity of these solutions were taken into consideration, such as polymer concentration, degree of hydrolysis, molecular weight, temperature, shear rate, and brine cation concentration. The only method used to estimate the unmeasured viscosity of a polymer solution in EOR was the Carreau model with Lee correlation, however, under conditions of low salinity and high molecular weight of the injection polymers, the correlation is not accurate. Thus, three ANN models were created (one for each evaluated polymer) and it was found that the model estimated viscosity values similar to those experimentally measured, being more accurate than the Carreau-Lee method. Thus, the application of artificial intelligence to estimate the viscosity of polymeric solutions can be considered a fast, reliable, and promising method to improve the EOR process.

Rostami et al. (2018b) also used artificial intelligence to estimate the viscosity of PHPA solutions from neural network models. It can be seen that the models can be promising to estimate viscosity values of polymeric solutions because they have high precision and reliability. Furthermore, it was found that temperature was the most influential parameter in the models, with a negative effect on the viscosity of the PHPA solution.

Corredor-Rojas et al. (2018) developed a Multilayer Perceptron (MLP) neural network model to predict the viscosities of PHPA-based nanopolymer systems and xanthan gum nanosystems. MLP is an artificial intelligence computer system applied to find complex correlations between inputs and outputs of a system, comprising a network model that includes several layers, the inputs being assigned to the first layer, the output to the last layer, and the relationships between input-output layers are called hidden layers. In this system, each layer has a determined number of neurons, with the number of neurons in the first layer corresponding to the number of input variables, hidden layers have neurons defined by trial-error and only one neuron for the last layer, corresponding to the output of the model. To obtain an efficient neural network, different numbers of neurons were considered and the system was tested with Bayesian Regularization (BR), 
Table 5. Summary of the main activities involving artificial intelligence.

\begin{tabular}{|c|c|c|}
\hline Year & Activity & Authors \\
\hline $2018 \mathrm{a}$ & $\begin{array}{l}\text { Developed a multi-layer feed-forward neural network algorithm to estimate the } \\
\mathrm{CO}_{2} \text { adsorption equilibrium in activated carbon }\end{array}$ & Rostami et al. \\
\hline 2016 & $\begin{array}{l}\text { Studied an innovative Least Square Support Vector Machine (LS-SVM) } \\
\text { algorithm to create a model capable of predicting the effective thermal } \\
\text { conductivity in dry sandstones }\end{array}$ & Rostami et al. \\
\hline $2017 \mathrm{a}$ & $\begin{array}{l}\text { Used the Genetic Programming (GP) method to estimate the interfacial } \\
\text { tension between hydrocarbon/water in reservoirs }\end{array}$ & Rostami et al. \\
\hline $2017 \mathrm{c}$ & $\begin{array}{l}\text { Performed a GP as a mathematical strategy to estimate the thermal } \\
\text { conductivity of supercritical } \mathrm{CO}_{2}\end{array}$ & Rostami et al. \\
\hline $2017 \mathrm{~b}$ & $\begin{array}{l}\text { Studied a GEP method to estimate the solubility of } \mathrm{CO}_{2} \text { in processes of } \mathrm{CO}_{2} \\
\text { flooding in oil reservoirs }\end{array}$ & Rostami et al. \\
\hline 2017 & $\begin{array}{l}\text { Developed a comparative study on the estimates of interfacial tension between } \\
\mathrm{CO}_{2} \text {-brine from the GEP, LS-SVM, and DT modeling methods with the ANN }\end{array}$ & Kamari et al. \\
\hline 2010 & $\begin{array}{l}\text { Applied the artificial neural network model to estimate the minimum amount } \\
\text { of PVA needed to improve the polymer flooding process. }\end{array}$ & Rezaian et al. \\
\hline 2016 & $\begin{array}{l}\text { Studied artificial neural network models to estimate the viscosity of polymer } \\
\text { solutions of three commercial acrylamide-based polymers }\end{array}$ & Kang et al. \\
\hline $2018 b$ & $\begin{array}{l}\text { Used artificial intelligence to estimate the viscosity of PHPA solutions from } \\
\text { neural network models. }\end{array}$ & Rostami et al. \\
\hline 2018 & $\begin{array}{l}\text { Developed a MLP neural network model to predict the viscosities of } \\
\text { PHPA-based nanopolymer systems and xanthan gum nanosystems }\end{array}$ & Corredor-Rojas et al. \\
\hline
\end{tabular}

Levenberg-Marquardt (LM), Resilient Backpropagation (RB), and Scaled Conjugate Gradient (SCG) algorithms, which consist of different methods to train MLP networks from real data. Thus, it was found that the BR-MLP model was the most promising among the others to estimate the viscosity of nanopolymeric systems, presenting an $R^{2}$ of 0.999 and an error of $2.46 \%$. The BR-MLP neural network model can be considered highly accurate to estimate viscosity under different conditions of shear, temperature, polymer type, concentration of nanoparticles and polymer, among others.

The summary of the main activities carried out involving the application of artificial intelligence and genetic programming is shown in Table 5 .

\section{Conclusion}

Among the chemical enhanced oil recovery methods, the injection of polymers as viscosifying agents stands out due to its efficiency and technical and economic feasibility. Several synthetic polymers and biopolymers can be used for this EOR technique, in particular application of partially hydrolyzed polyacrylamide, because of its good viscosifying property, abundant availability and low cost. However, PHPA has stability problems in reservoirs with severe salinity and temperature conditions. Thus, the use of Hydrophobically Modified Associative Polymers
(HMAPs), Thermo-Viscosifying Polymers (TVPs) and nano-hybrid systems have been widely studied.

This extensive bibliographic survey indicates that several factors influence the performance of HMAPs and TVPs, such as polymer concentration, type of chemical group inserted in the water-soluble polymer chain, salinity, temperature and shear rate, among others. In general, both HMAPs and TVPs can provide significant improvements in salt tolerance as well as temperature and shear resistance, increasing the oil recovery factor based on tests in porous media, among others. Furthermore, the application of nanoparticles for the production of hybrid nanopolymer systems can improve the performance of the injection polymers applied in EOR, increasing the viscosity of the systems and improving oil recovery.

In general, hydrophobically modified associative polymers and thermo-viscosifying polymers are promising alternatives for application as viscosifying agents for enhanced oil recovery, especially in harsh reservoir conditions (high salinity and temperature). In addition, the application of nanoparticles also is promising to improve the polymer flooding process.

This review depicts the evolution of studies of the injection of these polymer systems for EOR, indicating the importance of conducting further research in this area to find innovative solutions to improve oil recovery efficiency. Furthermore, it can be seen the promising applications of artificial intelligence and genetic programming in 
estimating important parameters for reservoir engineering and these methods can also be applied to estimate and optimize polymer flooding processes in enhanced oil recovery, through estimating values of viscosity of polymeric solutions and the prediction of the performance of these injection fluids in reservoirs.

Acknowledgments. We thank Brazil's National Council for Scientific and Technological Development (CNPq) for support.

\section{References}

Abdullahi M.B., Rajaei K., Junin R., Bayat A.E. (2019) Appraising the impact of metal-oxide nanoparticles on rheological properties of HPAM in different electrolyte solutions for enhanced oil recovery, J. Pet. Sci. Eng. 172, 1, 1057-1068.

Abidin A.Z., Puspasari T., Nugroho W.A. (2012) Polymers for enhanced oil recovery technology, Procedia Chem. 4, 1, 11-16.

Abirov R., Ivakhnenko A.P., Abirov Z., Eremin N.A. (2019) The associative polymer flooding: an experimental study, J. Pet. Explor. Prod. Technol. 1, 1, 1-8.

Afolabi R.O., Oluyemi G.F., Officer S., Ugwu J.O. (2019) Hydrophobically associating polymers for enhanced oil recovery - Part A: A review on the effects of some key reservoir conditions, J. Pet. Sci. Eng. 180, 1, 681-698.

Akbari S., Mahmood S.M., Tan I.M., Ghaedi H., Ling O.L. (2017a) Assessment of polyacrylamide based co-polymers enhanced by functional group modifications with regards to salinity and hardness, Polymers 9, 1, 647-662.

Akbari S., Mahmood S.M., Tan I.M., Ling O.L., Ghaedi H. (2017b) Effect of aging, antioxidant, and mono- and divalent ions at high temperature on the rheology of new polyacrylamide-based co-polymers, Polymers 9, 10, 480-493.

Amirian E., Dejam M., Chen Z. (2018) Performance forecasting for polymer flooding in heavy oil reservoirs, Fuel 216, 1, 83-100.

Azad M.S., Dalsania Y., Trivedi J.J. (2018) Understanding the flow behavior of copolymer and associative polymers in porous media using extensional viscosity characterization: effect of hydrophobic association, Can. J. Chem. Eng. 96, 11, 2498-2508.

Bai B., Zhou J., Yin M. (2015) A comprehensive review of polyacrylamide polymer gels for conformance control, Pet. Explor. Dev. 42, 4, 525-532.

Bai Y., Shang X., Wang Z., Zhao X. (2018) Experimental study on hydrophobically associating hydroxyethyl cellulose flooding system for enhanced oil recovery, Energy Fuels. 32, 6, 6713-6725.

Buckley S.E., Leverett M.C. (1942) Mechanism of fluid displacement in sands, Trans. 146, 1, 107-116.

Cao P., Mangadlao J.D., Advincula R.C. (2015) Stimuliresponsive polymers and their potential application in oil-gas industry, Polym. Rev. 55, 4, 706-733.

Chen Q., Wang Y., Lu Z., Feng Y. (2013) Thermo-viscosifying polymer used for enhanced oil recovery: rheological behaviors and core flooding test, Polym. Bull. 70, 2, 391-401.

Cheraghian G. (2016) Effect of nano titanium dioxide on heavy oil recovery during polymer flooding, Petrol. Sci. Tech. 34, 7, 633-641.

Cheraghian G., Hendraningrat L. (2016) A review on applications of nanotechnology in the enhanced oil recovery part A: effects of nanoparticles on interfacial tension, Int. Nano Lett. 6, 2, 129-138.
Choi B., Jeong M.S., Lee K.S. (2014) Temperature-dependent viscosity model of PHPA polymer through high-temperature reservoirs, Polym. Degrad. Stab. 110, 1, 225-231.

Choi B., Yu K., Lee K.S. (2016) Modelling of polymer retention during low concentrated PHPA polymer flooding in the heterogeneous reservoirs, Int. J. Oil, Gas Coal Technol. 11, 3, 249-263.

Clark A., Howe A.M., Mitchell J., Staniland J., Hawkes L.A. (2015) How viscoelastic-polymer flooding enhances displacement efficiency, in: SPE Asia Pacific Enhanced Oil Recovery Conference, Soc. Pet. Eng, Malásia, pp. 675-687.

Corredor L.M., Husein M.M., Maini B.B. (2019) Effect of hydrophobic and hydrophilic metal oxide nanoparticles on the performance of xanthan gum solutions for heavy oil recovery, Nanomaterials 9, 1, 94-106.

Corredor-Rojas L.M., Hemmati-Sarapardeh A., Husein M.M., Dong M., Maini B.B. (2018) Rheological behavior of surface modified silica nanoparticles dispersed in partially hydrolyzed polyacrylamide and xanthan gum solutions: experimental measurements, mechanistic understanding, and model development, Energy Fuels 32, 10, 10628-10638.

Dashtbesh N., Enchéry G., Noetinger B. (2021) A dynamic coarsening approach to immiscible multiphase flows in heterogeneous porous media, J. Pet. Sci. Eng. 201, 1, 1-14.

Data M.J., Milanesio J.M., Martini R., Strumia M. (2018) Synthesis techniques for polymers applied to enhanced oil recovery, MOJ Polym. Sci. 2, 1, 17-20.

Delamaide E., Zaitoun A., Renard G., Tabary R. (2014) Pelican Lake field: first successful application of polymer flooding in a heavy oil reservoir, Soc. Pet. Eng. 17, 3, 1-22.

Deng Q., Li H., Li Y., Cao X., Yang Y., Song X. (2014) Rheological properties and salt resistance of a hydrophobically associating polyacrylamide, Aust. J. Chem. 67, 10, 1396-1402.

Divers T., Al-Hashmi A.R., Al-Maamari R.S., Favero C. (2018) Development of thermo-responsive polymers for CEOR in extreme conditions: applicability to Oman oil fields, in: $S P E$ EOR Conference at Oil and Gas West Asia, Soc. Pet. Eng, Oman, pp. 1-15.

Dubin P., Strauss U.P. (1967) Hydrophobic hypercoiling in copolymers of maleic acid and alkyl vinyl ethers, J. Phys. Chem. A. 71, 8, 2757-2759.

El-Hoshoudy A.N., Desouky S.E.M., Elkady M.Y., Al-Sabagh A.M., Betiha M.A., Mahmoud S. (2017a) Hydrophobically associated polymers for wettability alteration and enhanced oil recovery - Article review, Egypt. J. Pet. 26, 3, 757-762.

El-Hoshoudy A.N., Desouky S.E.M., Alsabagh A.M., Betiha M. A., El-Kady M.Y., Mahmoud S. (2017b) Evaluation of solution and rheological properties for hydrophobically associated polyacrylamide copolymer as a promised enhanced oil recovery candidate, Egypt. J. Pet. 26, 3, 779-785.

El-Hoshoudy A.N., Desouky S.M., Gomaa S. (2019) Application of acrylates in enhanced oil recovery, J. New Develop. Chem. 2, 3, 1-17.

Ferreira V.H.S., Moreno R.B.Z.L. (2020) Experimental evaluation of low concentration scleroglucan biopolymer solution for enhanced oil recovery in carbonate, Oil Gas Sci. Technol. Rev. IFP Energies nouvelles 75, 61, 1-17.

Firozjaii A.M., Moradi S. (2018) Sensitive analysis and optimization of the effective parameters on ASP flooding compared to polymer flooding using CMG-STARS, J. Pet. Environ. Biotechnol. 9, 1, 361-365. 
Firozjaii A.M., Saghafi H.R. (2019) Review on chemical enhanced oil recovery using polymer flooding: fundamentals, experimental and numerical simulation, Petroleum 1, 1, 163-174.

Gao C.H. (2011) Scientific research and field applications of polymer flooding in heavy oil recovery, J. Pet. Explor. Prod. Technol. 1, 2, 65-70.

Gao C.H. (2013) Viscosity of partially hydrolyzed polyacrylamide under shearing and heat, J. Pet. Explor. Prod. Technol. 3, 3, 203-206.

Gbadamosi A.O., Junin R., Manan M.A., Yekeen N., Augustine A. (2019a) Hybrid suspension of polymer and nanoparticles for enhanced oil recovery, Polym. Bull. 76, 1, 6193-6230.

Gbadamosi A.O., Junin R., Manan M.A., Agi A., Yusuff A.S. (2019b) An overview of chemical enhanced oil recovery: recent advances and prospects, Int. Nano Lett. 9, 3, 171-202.

Ghoumrassi-Barr S., Aliouche D. (2016) A rheological study of xanthan polymer for enhanced oil recovery, J. Macromol. Sci. Phys. 55, 8, 793-809.

Giraldo L.J., Giraldo M.A., Llanos S., Maya G., Zabala R.D., Nassar N.N., Franco C.A., Alvarado V., Cortés F.B. (2017) The effects of $\mathrm{SiO}_{2}$ nanoparticles on the thermal stability and rheological behavior of hydrolyzed polyacrylamide based polymeric solutions, J. Pet. Sci. Eng. 159, 1, 841-852.

Gou S., Luo S., Liu T., Zhao P., He Y., Pan Q., Guo Q. (2015) A novel water-soluble hydrophobically associating polyacrylamide based on oleic imidazoline and sulfonate for enhanced oil recovery, New J. Chem. 39, 10, 7805-7814.

Guérillot D., Kadiri M., Trabelsi S. (2020) Buckley-Leverett theory for two-phase immiscible fluids flow model with explicit phase-coupling terms, Water 12, 11, 1-18.

Han X., Zhang G., Yu J., Chen Z., Kurnia I. (2018) As investigation of retention and unusually high apparent viscosity of hydrophobically associative polymer in porous media, in: SPE Improved Oil Recovery Conference, Soc. Pet. Eng, Oklahoma, pp. 1-10.

Haruna M.A., Nourafkan E., Hu Z., Wen D. (2019) Improved polymer flooding in harsh environments by free-radical polymerization and the use of nanomaterials, Energy Fuels 33, 2, $1637-1648$

Haruna M.A., Gardy J., Yao G., Hu Z., Hondow N., Wen D. (2020) Nanoparticle modified polyacrylamide for enhanced oil recovery at harsh conditions, Fuel. 268, 1, 117186-117204.

Hashmet M.R., Onur M., Tan I.M. (2014a) Empirical correlations for viscosity of polyacrylamide solutions with the effects of salinity and hardness, J. Dispers. Sci. Technol. 35, 4, 510-517.

Hashmet M.R., Onur M., Tan I.M. (2014b) Empirical correlations for viscosity of polyacrylamide solutions with the effects of temperature and shear rate, J. Dispers. Sci. Technol. 35, 12, 1685-1690.

Hourdet D., L'Alloret F., Audebert R. (1994) Reversible thermothickening of aqueous polymer solutions, Polymer $\mathbf{3 5}$, 12, 2624-2630.

Hu Z., Haruna M., Gao H., Nourafkan E., Wen D. (2017) Rheological properties of partially hydrolyzed polyacrylamide seeded by nanoparticles, Ind. Eng. Chem. Res. 56, 12, 34563463.

International Energy Agency (2019) Global energy $\& \mathrm{CO}_{2}$ status report, Available at https://www.iea.org/geco/oil/2019.

Jang H.Y., Zhang K., Chon B.H., Choi H.J. (2015) Enhanced oil recovery performance and viscosity characteristics of polysaccharide xanthan gum solution, J. Ind. Eng. Chem. 21, 1, $741-745$.
Jiang F., Pu W., Li Y., Du D. (2015) A double-tailed acrylamide hydrophobically associating polymer: synthesis, characterization, and solution properties, J. Appl. Polym. Sci. 132, 38, 42569-42578.

Jung J.C., Zhang K., Chon B.H., Choi H.J. (2013) Rheology and polymer flooding characteristics of partially hydrolyzed polyacrylamide for enhanced heavy oil recovery, J. Appl. Polym. Sci. 127, 6, 4833-4839.

Kamal M.S., Sultan A.S., Al-Mubaiyedh U.A., Hussein I.A. (2015a) Review on polymer flooding: rheology, adsorption, stability, and field applications of various polymer systems, Polym. Rev. 55, 3, 491-530.

Kamal M.S., Sultan A.S., Al-Mubaiyedh U.A., Hussein I.A., Feng Y. (2015b) Rheological properties of thermoviscosifying polymers in high-temperature and high-salinity environments, Can. J. Chem. Eng. 93, 7, 1194-1200.

Kamal M.S., Sultan A. (2017) Thermosensitive water soluble polymers: a solution to high temperature and high salinity reservoirs, in: SPE Kingdom of Saudi Arabia Annual Technical Symposium and Exhibition, Soc. Pet. Eng, Dammam, pp. 1-16.

Kamari A., Pournik M., Rostami A., Amirlatifi A., Mohammadi A.H. (2017) Characterizing the $\mathrm{CO}_{2}$-brine Interfacial Tension (IFT) using robust modeling approaches: a comparative study, J. Mol. Liq. 246, 1, 32-38.

Kang P., Lim J., Huh C. (2016) Artificial neural network model to estimate the viscosity of polymer solutions for enhanced oil recovery, Appl. Sci. 67, 188-202.

Karkevandi-Talkhooncheh A., Rostami A., HemmatiSarapardeh A., Ahmadi M., Husein M.M., Dabir B. (2018) Modeling minimum miscibility pressure during pure and impure $\mathrm{CO}_{2}$ flooding using hybrid of radial basis function neural network and evolutionary techniques, Fuel 220, 1, 270-282.

Khamis M.A., Omer O.A., Kinawy M.M. (2018) Predicting the optimum concentration of partially hydrolyzed polyacrylamide polymer in brine solutions for better oil recovery, experimental study, in: SPE Kingdom of Saudi Arabia Annual Technical Symposium and Exhibition, Soc. Pet. Eng, Dammam, pp. 1-17.

Lai N., Dong W., Ye Z., Dong J., Qin X., Chen W., Chen K. (2012) A water-soluble acrylamide hydrophobically associating polymer: synthesis, characterization, and properties as EOR chemical, J. Appl. Polym. Sci. 1294, 1888-1896.

Landoll L. (1985) Hydrophobically modified polymers, US n. 4529523A, 16 jul. 1985.

Leblanc T., Braun O., Thomas A., Divers T., Gailard N., Favero C. (2015) Rheological properties of stimuli-responsive polymers in solution to improve the salinity and temperature performances of polymer-based chemical enhanced oil recovery, in: SPE Asia Pacific Enhanced Oil Recovery, Soc. Pet. Eng., Kuala Lumpur, pp. $1-17$.

Leiting S., Zhongbin Y., Zhuo Z., Changjiang Z., Shanshan Z., Zhidong G. (2010) Necessity and feasibility of improving the residual resistance factor of polymer flooding in heavy oil reservoirs, Pet. Sci. 7, 2, 251-256.

Leiting S., Lei C., Zhongbin Y., Wei Z., Jian Z., Jie Y., Jianbo J. (2012) Effect of polymer solution structure on displacement efficiency, Pet. Sci. 9, 2, 230-235.

Levitt D., Jouenne S., Bondino I., Santanach-Carreras E., Bourrel M. (2013) Polymer flooding of heavy oil under adverse mobility conditions, in SPE Enhanced Oil Recovery Conference, Soc. Pet. Eng, Malásia, pp. 1-13.

Li X., Xu Z., Yin H., Feng Y., Quan H.O. (2017) Comparative studies on enhanced oil recovery: thermoviscosifying polymer versus polyacrylamide, Energy Fuels 31, 3, 2479-2487. 
Li X., Shu Z., Luo P., Ye Z. (2018) Associating polymer networks based on cyclodextrin inclusion compounds for heavy oil recover, J. Chem. 1, 1-9.

Liu P., Mu Z., Wang C., Wang Y. (2017a) Experimental study of rheological properties and oil displacement efficiency in oilfields for a synthetic hydrophobically modified polymer, Sci. Rep. 7, 1, 8791-8801.

Liu R., Pu W., Sheng J.J., Du D. (2017b) Star-like hydrophobically associative polyacrylamide for enhanced oil recovery: comprehensive properties in harsh reservoir condition, J. Taiwan Inst. Chem. Eng. 80, 1, 639-649.

Ma Q., Shuler P.J., Aften C.W., Tang Y. (2015) Theoretical studies of hydrolysis and stability of polyacrylamide polymers, Polym. Degrad. Stab. 121, 1, 69-77.

Mahran S., Attia A., Saha B. (2018) A review on polymer flooding in enhanced oil recovery under harsh conditions, in: 11th International Sustainable Energy \& Environmental Protection Conference, Paisley, Scotland, pp. 1-6.

Maia A.M.S., Borsali R., Balaban R.C. (2009) Comparison between a polyacrylamide and a hydrophobically modified polyacrylamide flood in a sandstone core, Mater. Sci. Eng. C. 29, 2, 505-509.

Manichand R.N., Let K.P.M.S., Gil L., Quillien B., Seright R.S. (2013) Effective propagation of PHPA solutions through the Tambaredjo reservoir during a polymer flood, Soc. Pet. Eng. 28, 4, 358-368.

Manrique E., Ahmadi M., Samani S. (2017) Historical and recent observations in polymer floods: an update review, $C T$ F-Cienc. Tecn. Fut. 6, 5, 17-48.

Mogollón J.L., Yomdo S., Salazar A., Dutta R., Bobula D., Dhodapkar P.K., Lokandwala T., Chandrasekar V. (2019) Maximizing a mature field value by combining polymer flooding, well interventions, and infill drilling, in: SPE Oil and Gas India Conference and Exhibition, Soc. Pet. Eng, Mumbai, pp. 1-21.

Moura M.R.V., Moreno R.B.Z.L. (2019) Concentration, brine salinity and temperature effects on xanthan gum solutions rheology, Appl. Rheol. 29, 1, 69-79.

Nguyen B.D., Ngo T.K., Bui T.H., Pham D.K., Dinh X.L., Nguyen P.T. (2015) The impact of graphene oxide particles on viscosity stabilization for diluted polymer solutions using in enhanced oil recovery at HTHP offshore reservoirs, Adv. Nat. Sci: Nanosci. Nanotechnol. 6, 1, 1-8.

Nwidee L.N., Theophilus S., Barifcani A., Sarmadivaleh M., Iglauer S. (2016) EOR processes, opportunities and technological advancements, in Chemical Enhanced Oil Recovery (cEOR), L. Romero-Zerón (ed), Nova Brunswick, IntechOpen, pp. $1-52$.

Oliveira P.F., Costa J.A., Oliveira L.F.S., Mota L.S., Oliveira L. A., Mansur C.R.E. (2019) Hydrolysis and thermal stability of partially hydrolyzed polyacrylamide in high-salinity environments, J. Appl. Polym. Sci. 13629, 47793-47803.

Patokina O.Y. (2015) Polymer flooding by hydrophobically associating polyacrylamide (technology of preparation. The study of the processes of stability and degradation), in: SPE Russian Petroleum Technology Conference, Soc. Pet. Eng, Moscow, pp. 1-14.

Peng F., Ke Y., He J., Lu S., Hu X. (2019) Big effects of small nanoparticles on hydrophobically modified polyacrylamide in an aqueous solution, J. Appl. Polym. Sci. 136, 16, 47269-47277.

Pope G.A. (2011) Recent developments and remaining challenges of enhanced oil recovery, Soc. Pet. Eng. 63, 7, 65-68.
Quan H., Hu Y., Huang Z., Wenmeng D. (2019) Preparation and property evaluation of a hydrophobically modified xanthan gum XG-C16, J. Dispers. Sci. Technol. 1, 1, 1-12.

Rangel I.R., Thompson R.L., Pereira R.G., Abreu F.L.B. (2012) Experimental investigation of the enhanced oil recovery process using a polymeric solution, J. Braz. Soc. Mech. Sci. Eng. 34, 3, 285-293.

Reichenbach-Klinke R., Zimmermann T., Stavland A., Strand D. (2018) Temperature-switchable polymers for improved oil recovery, in: SPE Norway One Day Seminar, Soc. Pet. Eng, Bergen, pp. 1-18.

Rellegadla S., Prajapat G., Agrawal A. (2017) Polymers for enhanced oil recovery: fundamentals and selection criteria, Appl. Microbiol. Biotechnol. 101, 11, 4387-4402.

Rellegadla S., Bairwa H.K., Kumari M.R., Prajapat G., Nimesh S., Pareek N., Jain S., Agrawal A. (2018) An effective approach for enhanced oil recovery using nickel nanoparticles assisted polymer flooding, Energy Fuels 32, 11, 1121211221.

Rezaei A., Abdi M., Mohebbi A., Tatar A., Mohammadi A.H. (2016) Using surface modified clay nanoparticles to improve rheological behavior of hydrolized polyacrylamid (HPAM) solution for enhanced oil recovery with polymer flooding, $J$. Mol. Liq. 222, 1, 1148-1156.

Rezaian A., Kordestany A., Sefat M.H. (2010) Experimental and artificial neural network approaches to predict the effect of PVA (Poly Vinyl Acetate) on the rheological properties of water and crude oil in EOR processes, in: Nigeria Annual International Conference and Exhibition, Soc. Pet. Eng, Nigeria, pp. 1-6.

Rostami A., Anbaz M.A., Gahrooei H.R.E., Arabloo M., Bahadori A. (2018a) Accurate estimation of CO2 adsorption on activated carbon with Multi-Layer Feed-Forward Neural Network (MLFNN) algorithm, Egypt. J. Pet. 27, 1, 65-73.

Rostami A., Arabloo M., Ebadi H. (2017a) Genetic programming (GP) approach for prediction of supercritical $\mathrm{CO}_{2}$ thermal conductivity, Chem. Eng. Res. Des. 122, 1, 164-175.

Rostami A., Arabloo M., Kamari A., Mohammadi A.H. (2017b) Modeling of $\mathrm{CO}_{2}$ solubility in crude oil during carbon dioxide enhanced oil recovery using gene expression programming, Fuels 210, 1, 768-782.

Rostami A., Ebadi H., Arabloo M., Meybodi M.K., Bahadori A. (2017c) Toward Genetic Programming (GP) approach for estimation of hydrocarbon/water interfacial tension, J. Mol. Liq. 230, 1, 175-189.

Rostami A., Kalantari-Meybodi M., Karimi M., Tatar A., Mohammadi A.H. (2018b) Efficient estimation of hydrolyzed polyacrylamide (HPAM) solution viscosity for enhanced oil recovery process by polymer flooding, Oil Gas Sci. Technol. Rev. IFP Energies Nouvelles 73, 22, 1-17.

Rostami A., Masoudi M., Ghaderi-Ardakani A., Arabloo M., Amani M. (2016) Effective thermal conductivity modeling of sandstones: SVM framework analysis, Int. J. Thermophys. 37, 6, 59-73.

Roy D., Brooks W.L.A., Sumerlin B.S. (2013) New directions in thermoresponsive polymers, Chem. Soc. Rev. 42, 17, $7214-7243$

Sarsenbekuly B., Kang W., Fan H., Yang H., Dai C., Zhao B., Aidarova S.B. (2017a) Study of salt tolerance and temperature resistance of a hydrophobically modified polyacrylamide based novel functional polymer for EOR, Colloids Surf. A Physicochem. Eng. Asp. 514, 1, 91-97. 
Sarsenbekuly B., Kang W., Yang H., Zhao B., Aidarova S., Yu B., Issakhov M. (2017b) Evaluation of rheological properties of a novel thermo-viscosifying functional polymer for enhanced oil recovery, Colloids Surf. A Physicochem. Eng. Asp. 532, 1, 405-410.

Sedaghat M.H., Hatampour A., Razmi R. (2013) Investigating the role of polymer type and dead end pores distribution on oil recovery efficiency during ASP flooding, Egypt. J. Pet. 22, 2, 241-247.

Sheng J.J., Leonhardt B., Azri N. (2015) Status of polymerflooding technology, Soc. Pet. Eng. 54, 2, 1116-1126.

SNF (2020) A cost-effective EOR technique to reduce carbon intensity with polymer flooding and modular skids, Available in: https://www.snf.com/wp-content/uploads/2020/08/CostEfficient-EOR-3p-v4.pdf $>$. Access in: 19 may. 2021.

Stavland A., Jonsbraten H.C., Lohne A., Moen A., Giske N.H. (2010) Polymer flooding - Flow properties in porous media versus rheological parameters, in: SPE EUROPEC/EAGE Annual Conference and Exhibition, Soc. Pet. Eng, Barcelona, pp. 1-15.

Su X., Feng Y. (2018) Thermoviscosifying smart polymers for oil and gas production: state of the art, in: Chemphyschem. 19, 16, 1941-1955.

Thomas A., Gaillard N., Favero C. (2012) Some key features to consider when studying acrylamide-based polymers for chemical enhanced oil recovery, Oil Gas Sci. Technol. - Rev. IFP Energies Nouvelles 67, 6, 887-902.

Thomas A. (2016) Polymer flooding, in: Chemical Enhanced Oil Recovery (cEOR), L. Romero-Zerón (ed.), Nova Brunswick, IntechOpen, pp. 55-99.

Wang Y., Feng Y., Wang B., Lu Z. (2010) A novel thermoviscosifying water-soluble polymer: synthesis and aqueous solution properties, J. Appl. Polym. Sci. 116, 6, 3516-3524.

Wang Y., Lu Z., Han Y., Feng Y., Tang C. (2011) A novel thermoviscosifying water-soluble polymer for enhancing oil recovery from high-temperature and high-salinity oil reservoirs, Adv. Mat. Res. 306-307, 1, 654-657.

Wang G., Yi X., Feng X., Jing B., Ouyang J. (2012) Synthesis and study of a new copolymer for polymer flooding in hightemperature, high-salinity reservoirs, Chem. Technol. Fuels Oils 48, 2, 112-119.

Wang C., Liu P., Wang Y., Yuan Z., Xu Z. (2018a) Experimental study of key effect factors and simulation on oil displacement efficiency for a novel modified polymer BDHMHEC, Sci. Rep. 8, 1, 3860-3869.

Wang M., Sun G., Han P., Su X., Feng Y. (2018b) Thermoviscosifying polymers based on polyether prepared from inverse emulsion polymerization, J. Appl. Polym. Sci. 135, 39, 46696-46705.

Wei B., Romero-Zerón L. (2014) The evaluation of a technological trend in polymer flooding for heavy oil recovery, J. Pet. Sci. Eng. 32, 19, 2396-2404.

Wei B., Romero-Zerón L., Rodrigue D. (2014) Mechanical properties and flow behavior of polymers for enhanced oil recovery, J. Macromol. Sci. Phys. 53, 4, 625-644.

Wever D.A.Z., Picchioni F., Broekhuis A.A. (2011) Polymers for enhanced oil recovery: A paradigm for structure-property relationship in aqueous solution, Prog. Polym. Sci. 36, 11, $1558-1628$.

Wu Y., Liu X., Wang Y., Guo Z., Feng Y. (2012) Synthesis and aggregation behaviors of well-defined thermoresponsive pentablock terpolymers with tunable LCST, Macromol. Chem. Phys. 213, 14, 1489-1498.

Xie K., Cao B., Lu X., Jiang W., Zhang Y., Li Q., Song K., Liu J., Wang W., Lv J., Na R. (2019) Matching between the diameter of the aggregates of hydrophobically associating polymers and reservoir pore-throat size during polymer flooding in an offshore oilfield, J. Pet. Sci. Eng. 177, 1, 558-569.

Xu L., Xu G., Liu T., Chen Y., Gong H. (2013) The comparison of rheological properties of aqueous welan gum and xanthan gum solutions, Carbohydr. Polym. 92, 1, 516-522.

Yadav U.S., Kumar H., Roy V., Juyal S., Tripathi A., Shanker A. (2020) Experimental evaluation of partially hydrolyzed polyacrylamide and silica nanoparticles solutions for enhanced oil recovery, J. Pet. Explor. Prod. Technol. 10, 1, 1109-1114.

Zeyghami M., Kharrat R., Ghazanfari M.H. (2014) Investigation of the applicability of nano silica particles as a thickening additive for polymer solutions applied in EOR processes, Energ. Source Part A 36, 12, 1315-1324.

Zhang Z., Li J., Zhou J. (2011a) Microscopic roles of "viscoelasticity" in HPMA polymer flooding for EOR, Transp. Porous Media 86, 1, 199-214.

Zhang P., Wang Y., Chen W., Yu H., Qi Z., Li K. (2011b) Preparation and solution characteristics of a novel hydrophobically associating terpolymer for enhanced oil recovery, $J$. Solution Chem. 40, 3, 447-457.

Zhang R., Ye Z., Peng L., Qin N., Shu Z., Luo P. (2012) The shearing effect on hydrophobically associative water-soluble polymer and partially hydrolyzed polyacrylamide passing through wellbore simulation device, J. Appl. Polym. Sci. 127, 1, 682-689.

Zhang P., Wang Y., Yang Y., Chen W., Bai S. (2015) Effective viscosity in porous media and applicable limitations for polymer flooding of an associative polymer, Oil Gas Sci. Technol. - Rev. IFP Energies nouvelles 70, 6, 931-939.

Zhang Y., Feng Y., Li B., Han P. (2019) Enhancing oil recovery from low-permeability reservoirs with a self-adaptive polymer: a proof-of-concept study, Fuels 251, 1, 136-146.

Zheng C., Cheng Y., Wei Q., Li X., Zhang Z. (2017) Suspension of surface-modified nano- $\mathrm{SiO}_{2}$ in partially hydrolyzed aqueous solution of polyacrylamide for enhanced oil recovery, Colloids Surf. A Physicochem. Eng. Asp. 524, 1, 169-177.

Zhong C., Luo P., Ye Z., Chen H. (2009) Characterization and solution properties of a novel water-soluble terpolymer for enhanced oil recovery, Polym. Bull. 62, 1, 79-89.

Zhong H., Li Y., Zhang W., Yin H., Lu J., Guo D. (2018) Microflow mechanism of oil displacement by viscoelastic hydrophobically associating water-soluble polymers in enhanced oil recovery, Polymers 10, 6, 628-642.

Zhou W., Zhang J., Han M., Xiang W., Feng G., Jiang W. (2007) Application of hydrophobically associating watersoluble polymer for polymer flooding in China offshore heavy oilfield, in: International Petroleum Technology Conference, Soc. Pet. Eng, Dubai, pp. 1-5.

Zhu Y., Xu Y., Huang G. (2013) Synthesis and aqueous solution properties of novel thermosensitive polyacrylamide derivatives, J. Appl. Polym. Sci. 1302, 766-775.

Zhu D., Han Y., Zhang J., Li X., Feng Y. (2014a) Enhancing rheological properties of hydrophobically associative polyacrylamide aqueous solutions by hybriding with silica nanoparticles, J. Appl. Polym. Sci. 131, 19, 40876-40883.

Zhu D., Wei L., Wang B., Feng Y. (2014b) Aqueous hybrids of silica nanoparticles and hydrophobically associating hydrolyzed polyacrylamide used for EOR in high-temperature and high-salinity reservoirs, Energies 7, 6, 3858-3871.

Zou C., Zhao P., Hu X., Yan X., Zhang Y., Wang X., Song R., Luo P. (2013) $\beta$-Cyclodextrin-functionalized hydrophobically associating acrylamide copolymer for enhanced oil recovery, Energy Fuels 27, 5, 2827-2834. 